\title{
Cryptic inoviruses revealed as pervasive in bacteria and archaea across Earth's biomes
}

\author{
Simon Roux ${ }^{1 \star}$, Mart Krupovic ${ }^{2}{ }^{2}$, Rebecca A. Daly ${ }^{3}$, Adair L. Borges ${ }^{4}$, Stephen Nayfach', \\ Frederik Schulz ${ }^{1}{ }^{1}$, Allison Sharrar ${ }^{5}$, Paula B. Matheus Carnevali $\mathbb{D}^{5}$, Jan-Fang Cheng1, \\ Natalia N. Ivanova ${ }^{1}$, Joseph Bondy-Denomy ${ }^{4,6}$, Kelly C. Wrighton ${ }^{3}$, Tanja Woyke ${ }^{1}{ }^{1}$, Axel Visel ${ }^{1}$, \\ Nikos C. Kyrpides ${ }^{1}$ and Emiley A. Eloe-Fadrosh ${ }^{1 \star}{ }^{1 \star}$
}

Bacteriophages from the Inoviridae family (inoviruses) are characterized by their unique morphology, genome content and infection cycle. One of the most striking features of inoviruses is their ability to establish a chronic infection whereby the viral genome resides within the cell in either an exclusively episomal state or integrated into the host chromosome and virions are continuously released without killing the host. To date, a relatively small number of inovirus isolates have been extensively studied, either for biotechnological applications, such as phage display, or because of their effect on the toxicity of known bacterial pathogens including Vibrio cholerae and Neisseria meningitidis. Here, we show that the current 56 members of the Inoviridae family represent a minute fraction of a highly diverse group of inoviruses. Using a machine learning approach leveraging a combination of marker gene and genome features, we identified 10,295 inovirus-like sequences from microbial genomes and metagenomes. Collectively, our results call for reclassification of the current Inoviridae family into a viral order including six distinct proposed families associated with nearly all bacterial phyla across virtually every ecosystem. Putative inoviruses were also detected in several archaeal genomes, suggesting that, collectively, members of this supergroup infect hosts across the domains Bacteria and Archaea. Finally, we identified an expansive diversity of inovirus-encoded toxin-antitoxin and gene expression modulation systems, alongside evidence of both synergistic (CRISPR evasion) and antagonistic (superinfection exclusion) interactions with co-infecting viruses, which we experimentally validated in a Pseudomonas model. Capturing this previously obscured component of the global virosphere may spark new avenues for microbial manipulation approaches and innovative biotechnological applications.

noviruses, bacteriophages from the Inoviridae family, exhibit unique morphological and genetic features. While the vast majority of known bacteriophages carry double-stranded DNA (dsDNA) genomes encapsidated into icosahedral capsids, inoviruses are instead characterized by rod-shaped or filamentous virions, circular single-stranded DNA genomes of $\sim 5-15 \mathrm{~kb}$ and a chronic infection cycle ${ }^{1-3}$ (Fig. 1a). Owing to their unique morphology and simple genome amenable to genetic engineering, several inoviruses are widely used for biotechnological applications, including phage display or as drug delivery nanocarriers ${ }^{4-7}$. Ecologically, cultivated inoviruses are known to infect hosts from only 5 bacterial phyla and 10 genera but can have significant effect on the growth and pathogenicity of their host ${ }^{8-10}$. For instance, an inovirus prophage, CTXphi, encodes and expresses the major virulence factor of toxigenic Vibrio cholerae ${ }^{11,12}$, whereas in other bacterial hosts, including Pseudomonas, Neisseria and Ralstonia, inovirus infections indirectly influence pathogenicity by altering biofilm formation and host colonization abilities ${ }^{8,13-16}$.

Despite these remarkable properties, their elusive life cycle and peculiar genomic and morphological properties have hampered systematic discovery of additional inoviruses: to date, only 56 inovirus genomes have been described ${ }^{17}$. Most inoviruses do not elicit negative effects on the growth of their hosts when cultivated in the laboratory and can thus easily evade detection. Furthermore, established computational approaches for the detection of virus sequences in whole-genome shotgun sequencing data are not efficient for inoviruses because of their unique and diverse gene content $^{18-20}$ (Fig. 1b). Finally, inoviruses are probably undersampled in viral metagenomes due to their long, flexible virions with low buoyant density ${ }^{21,22}$.

Here, we unveil a substantial diversity of 10,295 inovirus sequences, derived from a broad range of bacterial and archaeal hosts, and identified through an exhaustive search of 56,868 microbial genomes and 6,412 shotgun metagenomes using a custom computational approach to identify putative inovirus genomes. These sequences reveal that inoviruses are far more widespread, diverse and ecologically pervasive than previously appreciated, and provide a robust foundation to further characterize their biology across multiple hosts and environments.

\section{Results}

Inoviruses are highly diverse and globally prevalent. To evaluate the global diversity of inoviruses, an analysis of all publicly available inovirus genomes was first conducted to identify characteristic traits that would enable automatic discovery of divergent inovirus sequences (Supplementary Table 1). Across the 56 known Inoviridae genomes, the gene encoding the morphogenesis (pI) protein, an ATPase of the FtsK-HerA superfamily, represented the only

'DOE Joint Genome Institute, Walnut Creek, CA, USA. ${ }^{2}$ Department of Microbiology, Institut Pasteur, Paris, France. ${ }^{3}$ Department of Soil and Crop Sciences, Colorado State University, Fort Collins, CO, USA. ${ }^{4}$ Department of Microbiology and Immunology, University of California, San Francisco, San Francisco, CA, USA. ${ }^{5}$ Department of Earth \& Planetary Sciences, University of California, Berkeley, Berkeley, CA, USA. ${ }^{6}$ Quantitative Biosciences Institute, University of California, San Francisco, San Francisco, CA, USA. *e-mail: sroux@lbl.gov; eaeloefadrosh@lbl.gov 
a

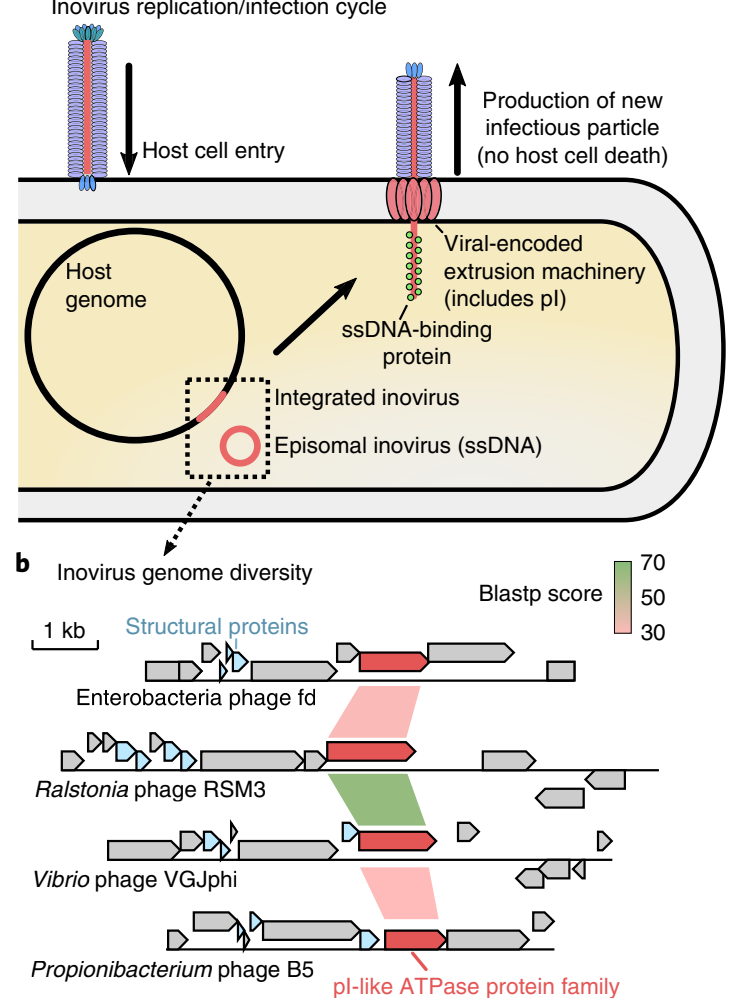

C Inovirus sequence identification approach

Step 1. pl-like ATPase detection (hmmsearch, score $\geq 30$ )

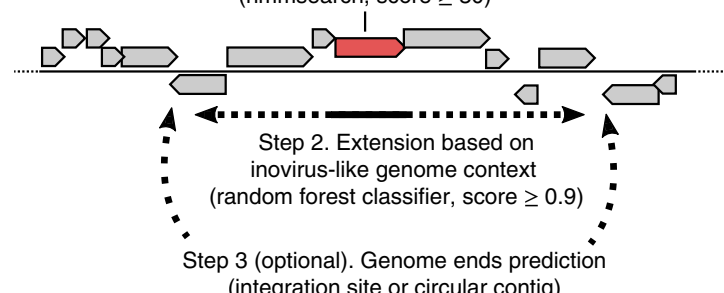

(integration site or circular contig)
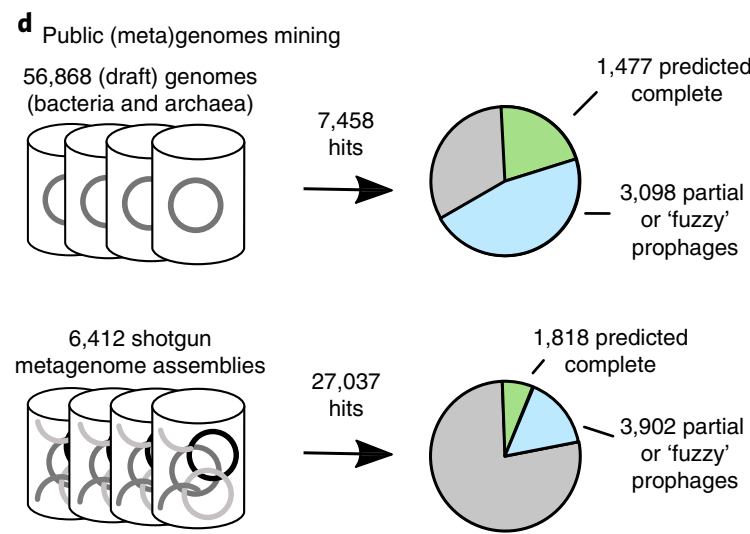

Fig. 1 | Overview of inovirus infection cycle, diversity and sequence detection process. a, Schematic of the inovirus persistent infection cycle and virion production. Inovirus genomes and particles are not to scale relative to the host cell and genome. ssDNA, single-stranded DNA. b, Comparison of selected inovirus genomes from isolates. The pl-like genes (the most conserved genes) are coloured in red, and sequence similarity between these genes (based on blastp) is indicated with coloured links between genomes. Putative structural proteins that can be identified based on characteristic features (gene length and presence of a TMD) are coloured in blue. Other genes are coloured in grey. c, Representation of the custom inovirus detection approach. The pl-like ATPase gene is coloured in red and other genes are coloured in grey. Dotted arrows indicate the region around pl-like genes that were searched for signs of an inovirus-like genome context and attachment site (see Supplementary Notes). d, Results of the search for inovirus sequences in prokaryote genomes and assembled metagenomes, after exclusion of putative false positives through manual inspection of predicted pl proteins (see Supplementary Notes). Predictions for which genome ends could be identified are indicated in green, while predictions without clear ends (that is, partial genomes or 'fuzzy' prophages with no predicted att site) are in blue, adding up to 10,295 curated predictions in total. Sequences for which no inovirus genome could be predicted around the initial pl-like gene are in grey. See also Supplementary Figs. 1-3.

conserved marker gene (Fig. 1a,b and Supplementary Fig. 1). However, three additional features specific of inovirus genomes could be defined: (1) short structural proteins (30-90 amino acids) with a single predicted transmembrane domain (TMD; Supplementary Table 1), (2) genes either functionally uncharacterized or similar to other inoviruses, and (3) shorter genes than those in typical bacterial or archaeal genomes (Supplementary Fig. 2A). These features were used to automatically detect inovirus sequences through a two-step process (Fig. 1b). First, pI-like proteins are detected through a standard hidden Markov model (HMM)-based similarity search. Then, a random forest classifier trained on genomes of isolate inoviruses and manually curated prophages used these genome features to identify inovirus sequences from the background host genome. This approach yielded $92.5 \%$ recall and $99.8 \%$ precision on our manually curated reference set (Fig. 1c, Supplementary Fig. 2 and Supplementary Notes).

This detection approach was applied to 56,868 bacterial and archaeal genomes and 6,412 metagenomes publicly available from the Integrated Microbial Genomes (IMG) database ${ }^{23}$ (Supplementary Table 2). After manual curation of edge cases and removal of detections not based on a clear inovirus-like ATPase, a total of 10,295 sequences were recovered (Fig. 1d, Supplementary Fig. 3 and Supplementary Notes). From these, 5,964 distinct species were identified using genome-wide average nucleotide identity (ANI), and only 38 of these included isolate inovirus genomes. About onethird of these species (30\%) encoded an 'atypical' morphogenesis gene, with an amino-terminal instead of carboxy-terminal TMD (Supplementary Fig. 3). Although this atypical domain organization has been observed in four isolate species currently classified as inoviruses, some of these inovirus-like sequences might eventually be considered as entirely separate groups of viruses. Sequence accumulation curves did not reach saturation, highlighting the large diversity of inoviruses yet to be sampled (Supplementary Fig. 4).

Inovirus sequences were identified in $6 \%$ of bacterial and archaeal genomes $(3,609$ of 56,868$)$ and $35 \%$ of metagenomes $(2,249$ of 6,412$)$. More than half of the species $(n=3,675)$ were exclusively composed of sequences assembled from metagenomes. These revealed that inoviruses are found in every major microbial habitat whether aquatic, soil or human associated, and throughout the entire globe (Fig. 2 and Supplementary Notes). Hence, inoviruses are much more diverse than previously estimated and globally distributed.

Inoviruses infect a broad diversity of bacterial hosts. To examine the host range of these inoviruses, we focused on the 2,284 inovirus species directly associated with a host, that is, proviruses 

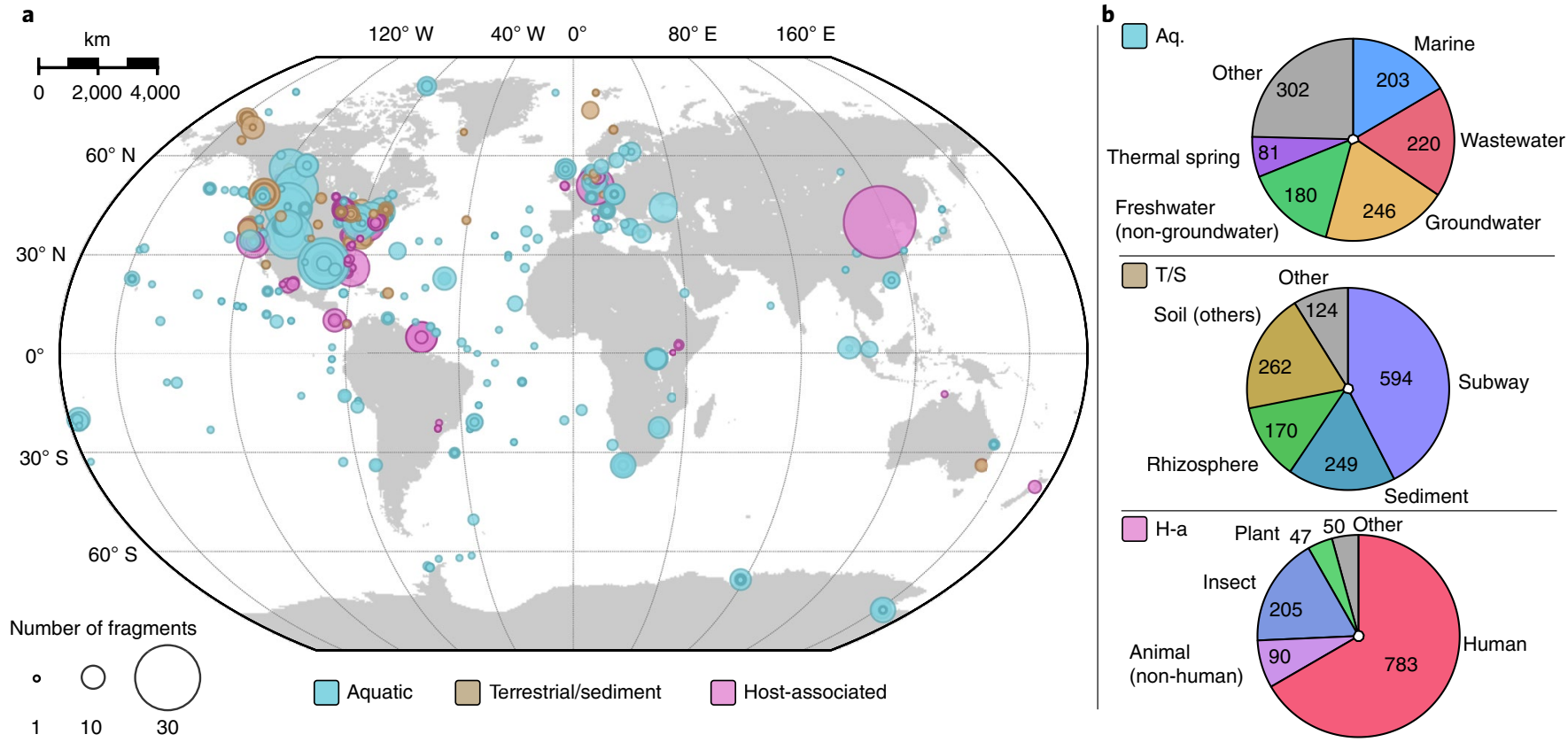

Fig. 2 | Geographical and biome distribution of inovirus sequences detected in metagenomes. a, Repartition of samples for which one or more inovirus sequence(s) was detected. Each sample is represented by a circle proportional to the number of inovirus detections and coloured according to their ecosystem type. $\mathbf{b}$, Breakdown of the number of inovirus detections by ecosystem subtype for each major ecosystem. A more detailed ecosystem distribution of each proposed inovirus family is presented in Supplementary Fig. 7. Aq., aquatic; $\mathrm{H}$-a, host-associated; T/S, terrestrial/sediment.

derived from a microbial genome (Fig. 3). The majority (90\%) of these species were associated with Gammaproteobacteria and Betaproteobacteria, from which most known inoviruses were previously isolated (Supplementary Table 1). However, the range of host genera within these groups was vastly expanded, including clinically and ecologically relevant microorganisms such as Azotobacter, Haemophilus, Kingella or Nitrosomonas (Supplementary Table 3 ). The remaining 412 species strikingly increased the potential host range of inoviruses to 22 additional phyla, including the Candidate Phyla Radiation (Fig. 3). For three of these (Acidobacteria, Chlamydiae and Spirochaetes), only short inovirus contigs were detected, lacking host flanking regions, which would provide confident host linkages. Hence, these contigs could potentially derive from sample contamination (for example, from reagents), and inovirus presence within these phyla remains uncertain (Supplementary Table 4). The notable host expansion is consistent with reported experimental observations of filamentous virus particles induced from a broad range of bacteria, for example, Mesorhizobium, Clostridium, Flavobacterium, Bacillus and Arthrobacter $^{24,25}$ (Fig. 3).

This large-scale detection of inovirus sequences in microbial genomes also enabled a comprehensive assessment of co-infection, both between different inoviruses and with other types of viruses. In the majority of cases, a single inovirus sequence was detected per genome, with multiple detections mostly found within Gammaproteobacteria, Betaproteobacteria and Spiroplasma genomes (Supplementary Fig. 5). Conversely, inovirus prophages were frequently detected along and sometimes colocalized with Caudovirales prophages, suggesting that these two types of phages frequently co-infect the same host cell (Supplementary Fig. 5 and Supplementary Notes). Overall, the broad range of bacteria and archaea infected by inoviruses combined with their propensity to co-infect a microbial cell with other viruses and their global distribution indicate that inoviruses probably play an important ecological role in all types of microbial ecosystems.
Inoviruses sporadically transferred from bacterial to archaeal hosts. Although no archaea-infecting inoviruses have been reported so arr $^{26}$, some inovirus sequences were associated with members of two archaeal phyla (Euryarchaeota and Aenigmarchaeota), which suggests that inoviruses infect hosts across the entire prokaryotic diversity (Fig. 3). These putative archaeal proviruses encoded the full complement of genes expected in an active inovirus (Fig. $4 \mathrm{a}$ and Supplementary Notes). Using PCR, we further confirmed the presence of a circular, excised form of the complete inovirus genome for the provirus identified in the Methanolobus profundi MobM genome (Fig. 4b, Supplementary Fig. 6 and Supplementary Notes). This indicates that our predictions in archaeal genomes are probably genuine inoviruses.

Few groups of viruses include both bacteriophages and archaeoviruses. Such evolutionary relationships between viruses infecting hosts from different domains of life might signify either descent from an ancestral virus that infected the common ancestor of bacteria and archaea, or horizontal virus transfer from one host domain to the other ${ }^{26-28}$. Here, the four archaea-associated inoviruses were clearly distinct from most other inoviruses and clustered only with metagenomic sequences in pI phylogeny (Fig. 4c). In addition, they were classified into two different proposed families (see below) corresponding to the two host groups, reflecting clear differences in their gene content (Fig. 4a,c and Supplementary Notes). The high genetic diversity of these archaea-associated inoviruses, combined with the lack of similarity to bacteria-infecting species, suggest that they are not derived from a recent host switch event.

A possible scenario would involve an ancestral group of inoviruses infecting the common ancestor of archaea, as postulated for the double-jelly-roll virus lineage ${ }^{28}$. However, to be confirmed, this hypothesis would require the detection of additional inoviruses in other archaeal clades or an explanation as to why inoviruses were retained only in a handful of archaeal hosts. Instead, on the basis of the current data, a more likely scenario involves ancient and rare events of interdomain inovirus transfer from bacteria to archaea, 


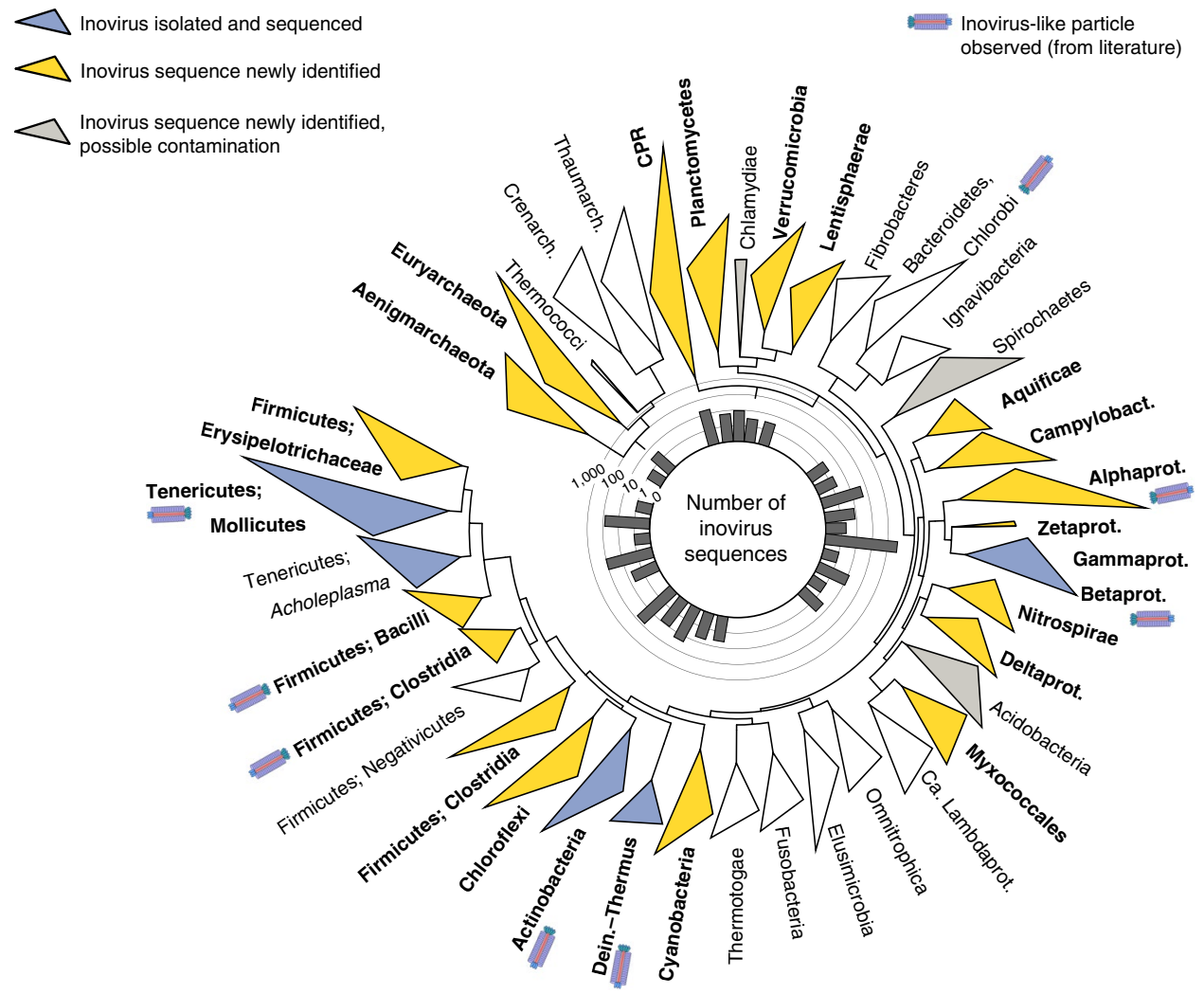

Tree scale: $0.1 \mapsto$

Fig. 3 | Phylum-wide distribution of inovirus detections across microbial genomes. The bacteria and archaea phylogenetic trees were computed based on 56 universal marker proteins. Monophyletic clades representing a single phylum (or class for proteobacteria) were collapsed when possible, and only clades including $\geq 30$ genomes or associated with an inovirus(es) are displayed. Clades for which one or more inovirus has been isolated and sequenced are coloured in blue, and clades that have not been previously associated with inovirus sequences are coloured in yellow. Clades for which inovirus-like particles had been reported and/or induced are indicated with a filamentous particle symbol. Putative host clades for which inovirus detection might result from sample contamination, that is, no clear host linkage based on an integrated prophage(s) or CRISPR spacer hit(s), are coloured in grey (Supplementary Table 4). Clades robustly associated with inoviruses in this study (that is, one or more detection unlikely to result from sample contamination) are highlighted in bold. The histogram at the centre indicates the total number of inovirus for each clade, on a $\log _{10}$ scale. Alphaprot., Alphaproteobacteria; Betaprot., Betaproteobacteria; Ca. Lambdaprot.; 'Candidatus Lambdaproteobacteria'; Campylobact., Campylobacterota; CPR, Candidate Phyla Radiation; Creanarch., Crenarchaeota; Dein.-Thermus, Deinococcus-Thermus; Deltaprot., Deltaproteobacteria; Gammaprot., Gammaproteobacteria; Thaumarch., Thaumarchaeota; Zetaprot., Zetaproteobacteria. See also Supplementary Figs. 4 and 5.

including possibly to a Methanosarcina host for which substantive horizontal transfers of bacterial genes have already been reported ${ }^{29}$.

Gene content classification reveals six distinct inovirus families. The vast increase of inovirus sequences provided a great opportunity for re-evaluation of the inovirus classification and the development of an expanded taxonomic framework for the large number of inovirus species identified. Similar to other bacterial viruses, especially temperate phages $^{30}$, inovirus genomes display modular organization and are prone to recombination and horizontal gene transfers ${ }^{31}$ (Supplementary Fig. 7). Hence, we opted to apply a bipartite network approach, in which genomes are connected to gene families, enabling a representation and clustering of the diversity based on shared gene content. A similar approach has been previously employed for the analysis of DNA and RNA viruses, and was shown to be efficient in cases in which the genomes to be clustered share only a handful of genes ${ }^{26,32-34}$. Here, this approach yielded 6 distinct groups of genomes divided into 212 subgroups (Fig. 5a and Supplementary Table 3).

A comparison of marker gene conservation between these groups and established viral taxa suggested that the former Inoviridae family should be reclassified as an order, provisionally divided into 6 candidate families and 212 candidate subfamilies, with few shared genes across candidate families (Fig. 5a, Supplementary Fig. 7 and Supplementary Notes). Beyond gene content, these proposed families also displayed clearly distinct host ranges as well as specific genome features, particularly in terms of genome size and coding density (Supplementary Fig. 7). Thus, we propose to establish these as candidate families named 'Protoinoviridae', 'Vespertilinoviridae', 'Amplinoviridae', 'Paulinoviridae', 'Densinoviridae' and 'Photinoviridae', on the basis of their isolate members and characteristics (see Supplementary Notes). If confirmed, and compared with currently recognized inoviruses, the genomes reported here would increase diversity by 3 families and 198 subfamilies.

The host envelope organization seems to play an important role in the evolution of inoviruses, which is reflected in their classification: members of the 'Protoinoviridae' and 'Amplinoviridae' are associated with diderm hosts-that is, Gram-negative bacteria with an outer membrane-whereas the other candidate families are associated with monoderm hosts or hosts without a cell wall (Supplementary Fig. 7). Conversely, no structuring by biome was observed and all proposed families were broadly detected across multiple types of ecosystems. Hence, we propose here a classification of inovirus diversity into six families based on gene content with coherent host ranges and 




Sp_2724278400_Ga0181639_109

原 Unclassified Candidatus Aenigmarchaeota

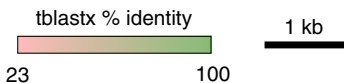

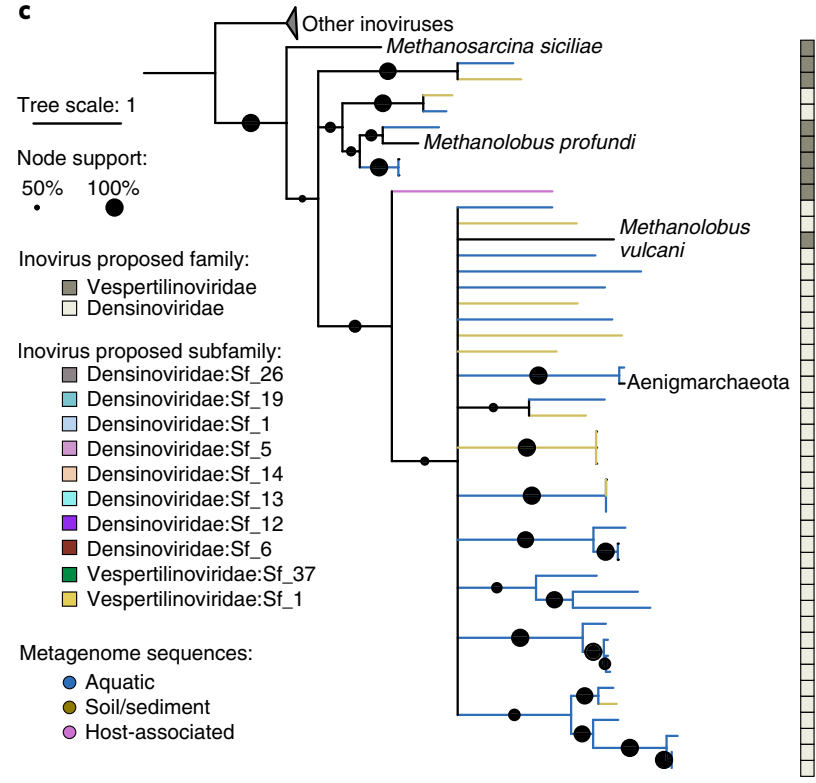

b Methanolobus profundi Mob M: Ga0073303_107

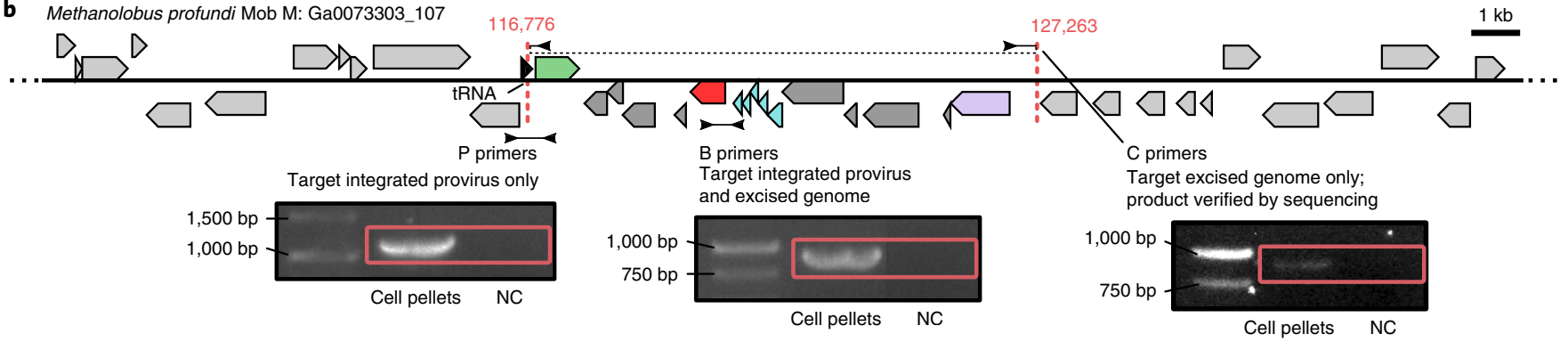

Fig. 4 | Characterization of archaea-associated inoviruses. a, Genome comparison of the four inovirus sequences detected in members of the Methanosarcinaceae family or Aenigmarchaeota candidate phylum. Genes are coloured according to their functional affiliation (light grey indicates ORFan). RC, sequence is reverse complemented. b, PCR validation of the predicted inovirus from the archaea host M. profundi MobM. Three primer pairs were designed and used to amplify across the predicted $5^{\prime}$ insertion site (P primers), within the predicted provirus (B primers) or across the junction of the predicted excised circular genome ( $C$ primers). The predicted provirus attachment site is indicated by dotted red lines along with corresponding genome coordinates. Products from C primers were sequenced and aligned to the $M$. profundi MobM genome to confirm that they spanned both ends of the provirus in the expected orientation and at the predicted coordinates (see Supplementary Notes and Supplementary Fig. 6). Red boxes indicate the expected product lengths. $P$ and $B$ primer amplifications were repeated twice, and the $C$ primer amplifications were repeated three times, with an identical result obtained for each replicate (Supplementary Fig. 11). NC, no template control. c, Phylogenetic tree of archaea-associated inoviruses and related sequences. The tree was built from pl protein multiple alignment with IQ-TREE. Nodes with support of <50\% were collapsed. Branches leading to inovirus species associated to a host are coloured in black, and the corresponding host is indicated on the tree. Branches leading to inovirus species assembled from metagenomes are coloured by type of environment. Classification of each inovirus species in proposed families and subfamilies is indicated next to the tree (see Fig. 5).

specific genomic features, which strongly suggests that they represent ecologically and evolutionarily meaningful units.

Inovirus genomes encode an extensive functional repertoire. The extended catalogue of inovirus genomes offers an unprecedented window into the diversity of their genes and predicted functions. Overall, 68,912 proteins were predicted and clustered into 3,439 protein families and 13,714 singletons. This is on par with the functional diversity observed in known Caudovirales genomes, the largest order of dsDNA viruses, for which the same number of proteins clustered into 12,285 protein families but only 8,552 singletons (see Methods). A putative function was predicted for 1,133 of the 3,439 inovirus protein families (iPFs). Most of these (>95\%) could be linked to virion structure, virion extrusion, DNA replication and integration, toxin-antitoxin systems or transcription regulation (Supplementary Table 5). A total of 51 and 47 distinct iPFs could be annotated as major and minor coat proteins, respectively, with an additional 934 iPFs identified as potentially structural based on their size and presence of a TMD (see Methods). Notably, each candidate inovirus family seemed to be associated with a specific set of structural proteins, including distinct major coat iPFs (Supplementary Fig. 8). Conversely, genome replication and integration-associated iPFs were broadly shared across candidate families (Fig. 5b). This confirms that replication-associated and integration-associated genes are among the most frequently exchanged among viral genomes and with other mobile genetic elements, especially in small single-stranded DNA viruses ${ }^{35}$.

In addition, 15 distinct sets of iPFs representing potential toxin-antitoxin pairs were identified across 181 inovirus genomes, including 10 unaffiliated iPFs that were predicted as putative antitoxins through co-occurrence with a toxin iPF (Fig. 5b and Supplementary Table 5; see Methods). These genes typically stabilize plasmids or prophages in host cell populations, although alternative roles in stress response and transcription regulation 
a

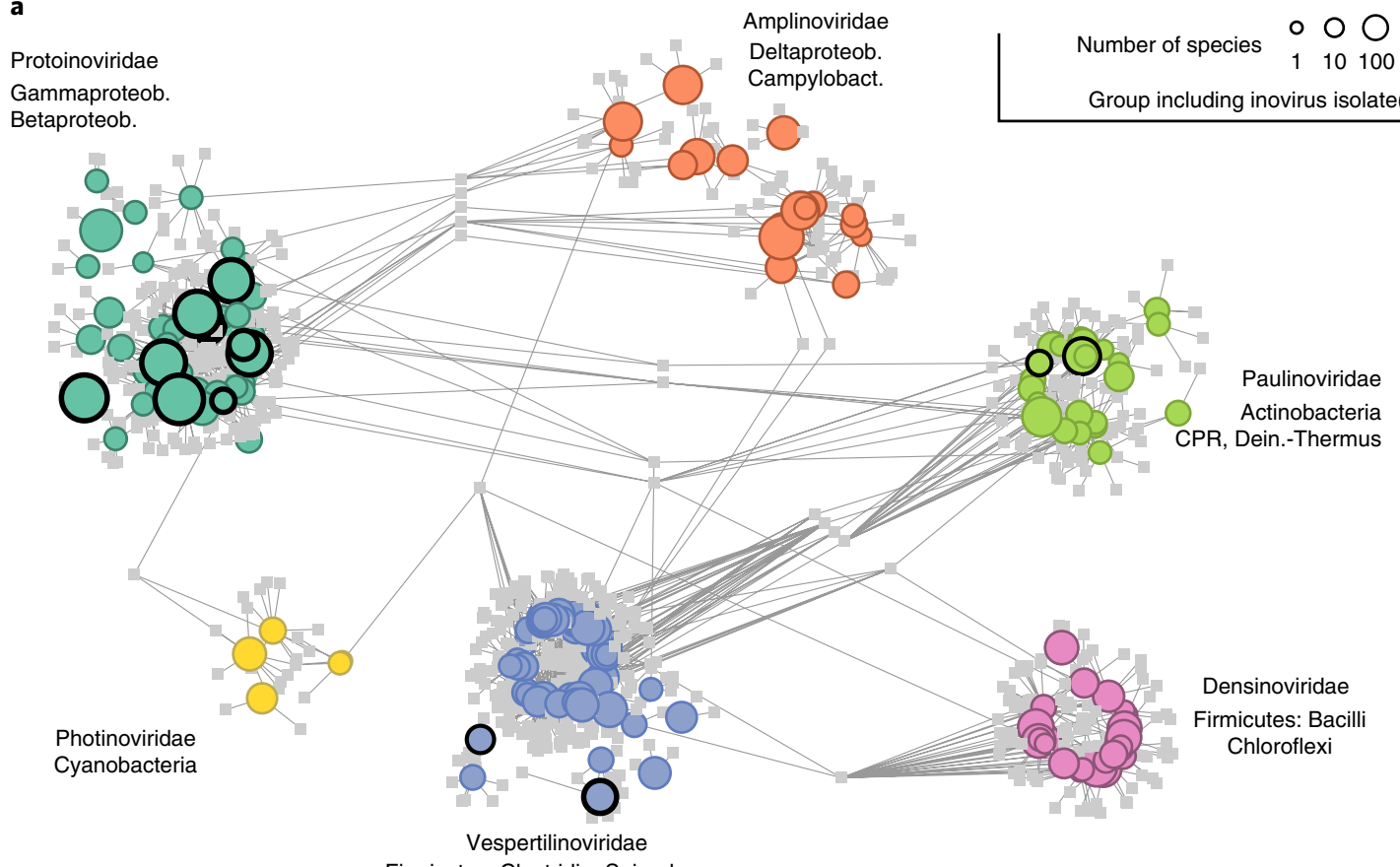

b

Firmicutes: Clostridia, Spiroplasma
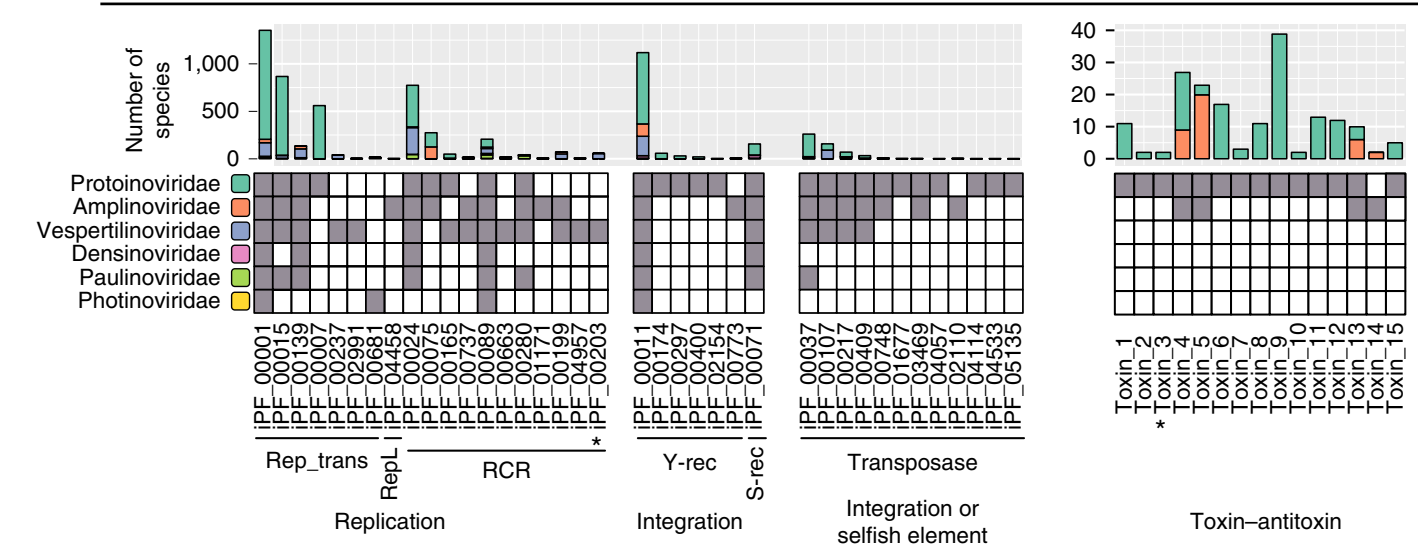

Fig. 5 | Inovirus genome sequence space and gene content. a, The bipartite network links genes represented as PCs in squares to proposed subfamilies represented as circles with a size proportional to the number of species in each candidate subfamily $\left(\log _{10}\right.$ scale), grouped and coloured by proposed family. Proposed subfamilies that include viral isolates are highlighted with a black outline. Candidate subfamilies are connected to PCs when $\geq 50 \%$ of the subfamily members contained this PC or $\geq 25 \%$ for the larger proposed subfamilies (see Methods). $\mathbf{b}$, Distribution of iPFs detected in two or more genomes, associated with genome replication, genome integration and toxin-antitoxin systems (see Supplementary Table 5). The presence of at least one sequence from an iPF (column) in a proposed family (row) is indicated with a grey square. Rolling circle replication (RCR) iPFs include only the RCR endonuclease motif, with the exception of iPF_00203 (highlighted with an asterisk), which also includes the C-terminal S3H motif typical of eukaryotic single-stranded DNA viruses. Transposases used by selfish integrated elements are indistinguishable from transposases domesticated by viral genomes using sequence analysis only; hence, these genes are gathered in a single 'integration or selfish element' category. All toxin-antitoxin pairs were predicted to be of type II, except for Toxin_3 (highlighted with an asterisk), which was predicted to be type IV. S-rec, serine recombinase; Y-rec, tyrosine recombinase. See also Supplementary Figs. 7-9.

have been reported ${ }^{36}$. In addition, toxin-antitoxin systems often affect host cell phenotypes, such as motility or biofilm formation ${ }^{1}$. Here, similar toxin proteins could be associated with distinct and seemingly unrelated antitoxins and vice versa, suggesting that gene shuffling and lateral transfer occur even within these tightly linked gene pairs (Supplementary Fig. 9). All but one toxin-antitoxin pairs were detected in proteobacteria-associated inoviruses, most likely because of a database bias. Thus, numerous uncharacterized iPFs across other candidate families of inoviruses may also encode previously undescribed toxin-antitoxin systems and, more generally, host manipulation mechanisms.
Inoviruses can both leverage and restrict co-infecting viruses. Finally, we investigated potential interactions between persistently infecting inoviruses, other co-infecting viruses, and the host clustered regularly interspaced short palindromic repeats (CRISPR)CRISPR-associated (Cas) immunity systems. CRISPR-Cas systems typically target bacteriophages, plasmids and other mobile genetic elements $^{37}$. We detected 1,150 inovirus-matching CRISPR spacers across 42 bacterial and 1 archaeal families. These spacers were associated with three types and eight subtypes of CRISPR-Cas systems, indicating that inoviruses are broadly targeted by antiviral defences (Fig. 6a, Supplementary Table 6 and Supplementary Notes). Several 


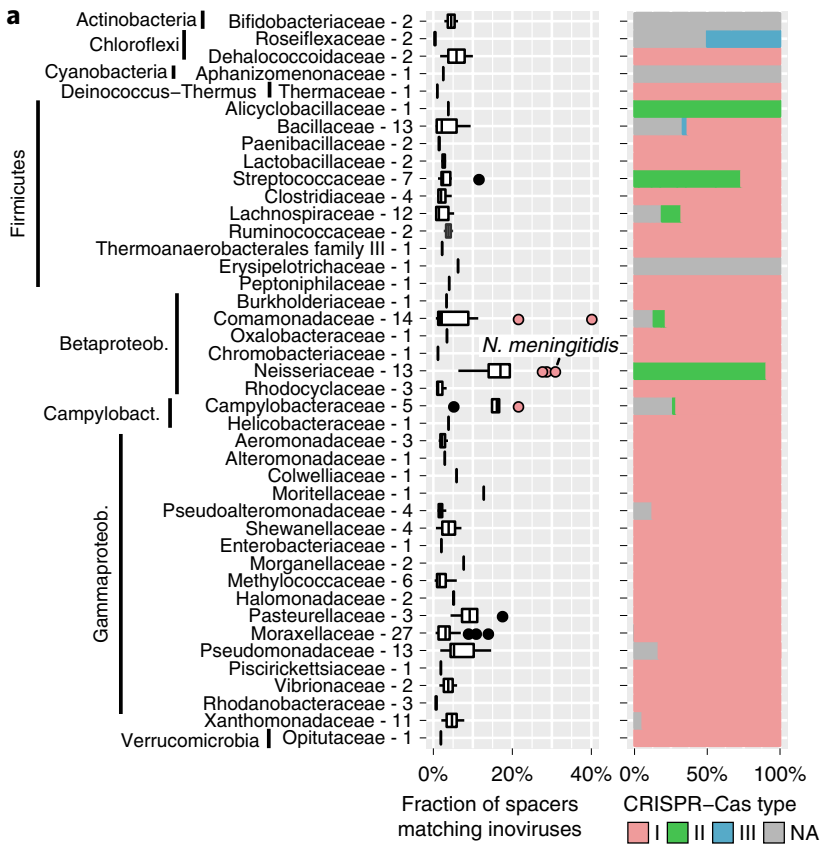

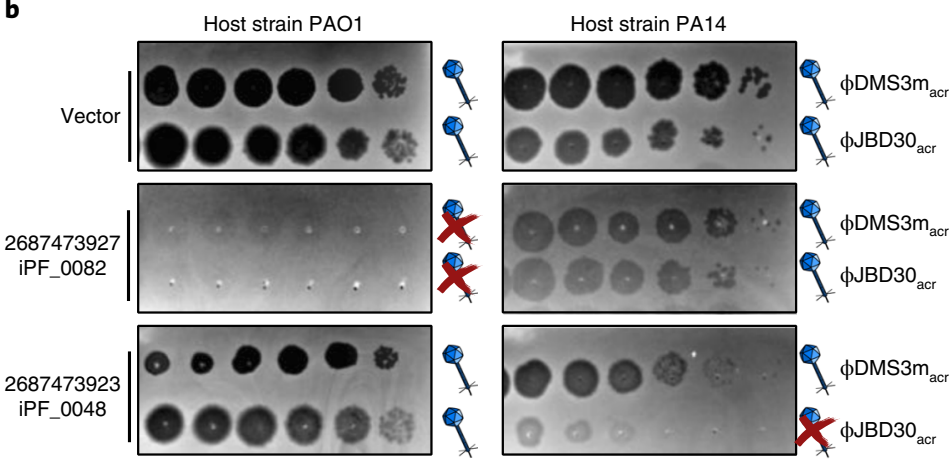

c

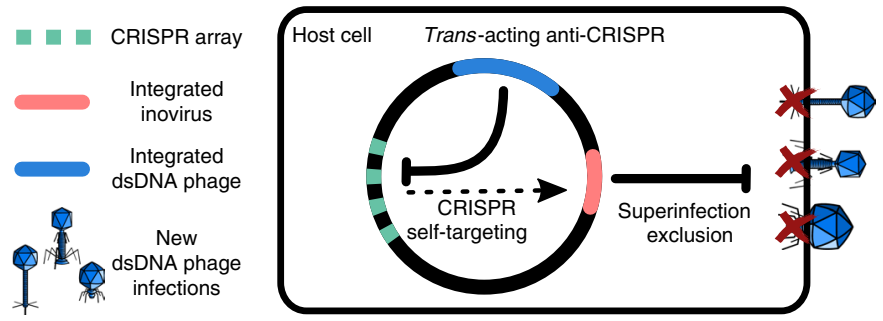

Fig. 6 | Interaction of inoviruses with CRISPR-Cas systems and co-infecting viruses. a, Proportion of the spacers matching an inovirus genome and the corresponding distribution of CRISPR-Cas systems. The proportions are calculated only on hosts with at least one spacer matching an inovirus sequence, with hosts grouped at the family rank (hosts unclassified at this rank were not included). In the boxplot, the lower and upper hinges correspond to the first and third quartiles, respectively, and the whiskers extend no further than \pm 1.5 times the interquartile range. Outliers identified as values larger than the third quartile plus three times the interquartile range from the complete distribution are highlighted in red. The number of observations is indicated next to each family. $\mathbf{b}$, Instances of superinfection exclusion observed when expressing individual inovirus genes in two P. aeruginosa strains: PAO1 and PA14. From top to bottom: cells were transformed with an empty vector, one expressing gene 2687473927 or one expressing gene 2687473923 . For each construct, host cells were challenged with serial dilutions (from left to right) of phages: $\phi J B D 30$ and $\phi D M S 3 \mathrm{~m}$. The formation of plaques (dark circles) indicates successful infection, whereas the absence of plaques indicates superinfection exclusion. Interpretation of infection outcome is indicated to the right of each lane, with successful infection represented by a phage symbol and superinfection exclusion represented by a phage symbol barred by a red cross. Results from additional superinfection exclusion experiments are presented in Supplementary Figs. 10 and 12. All superinfection experiments were conducted twice and produced similar results. c, Schematic representation of the possible mutualistic or antagonistic interactions between inovirus prophages (red) and co-infecting Caudovirales (blue). Mutualistic interactions include suppression of the CRISPR-Cas immunity, especially for integrated inoviruses targeted by the host cell CRISPR-Cas system ('self-targeting'). Antagonistic interactions primarily involve superinfection exclusion, in which a chronic inovirus infection prevents a secondary infection by an unrelated virus.

host groups, most notably Neisseria meningitidis, were clear outliers, that is, they displayed a particularly high ratio of inovirus-derived spacers suggesting a uniquely high level of spacer acquisition and inovirus infection (Fig. 6a). This is particularly notable because inoviruses were recently suggested to increase N.meningitidis pathogenicity ${ }^{13}$ and hints at conflicting host-inovirus interactions in this specific group.

Next, we examined instances of 'self-targeting', that is, CRISPR spacers matching an inovirus integrated in the same host genome. Among the 1,429 genomes that included both a CRISPR-Cas system and an inovirus prophage, only 45 displayed a spacer match(es) to a resident prophage (Supplementary Table 6), suggesting that self-targeting of these integrated elements is lethal and strongly counter-selected $^{38}$. This was confirmed experimentally using the Pseudomonas aeruginosa strain PA14 harbouring an integrated inovirus prophage (Pf1), for which the introduction of a plasmid carrying Pf1-targeting CRISPR spacers was lethal (Supplementary Fig. 10a). In the 45 cases of observed self-targeting, the corresponding CRISPR-Cas system is thus probably non-functional or inhibited via an anti-CRISPR (acr) locus, as recently described in dsDNA phages $^{38}$. We first evaluated ten hypothetical proteins, and hence candidate Acr proteins, from self-targeted inoviruses infecting P. aeruginosa; however, none showed Acr activity (Supplementary Notes and Supplementary Fig. 10b). Alternatively, inoviruses could leverage the Acr activity of a co-integrated virus. This hypothesis was further reinforced by the fact that 43 of the 45 self-targeted inoviruses were detected alongside co-infecting dsDNA phages, with 5 of these encoding known acr genes (Supplementary Table 6). We confirmed experimentally cross-protection by trans-acting Acr in the P.aeruginosa PA14 model, and observed that co-infection with an acr-encoding dsDNA bacteriophage rescued the lethality caused by self-targeted inoviruses (Supplementary Notes and Supplementary Fig. 10a).

While this represents an instance of beneficial co-infection for inoviruses, we also uncovered evidence of antagonistic interactions between inoviruses and dsDNA bacteriophages. Specifically, 2 of the 10 inovirus-encoded hypothetical proteins tested strongly limited infection of Pseudomonas cells by different bacteriophages (Fig. 6b, Supplementary Figs. 10c and 12 and Supplementary Notes). This superinfection exclusion effect was found to be host and virus strain dependent, which could drive intricate tripartite coevolution dynamics. Thus, these preliminary observations indicate that inoviruses may not only evade CRISPR-Cas immunity by leveraging the Acr activity of co-integrated phages, but also significantly influence the infection dynamics of unrelated co-infecting viruses through superinfection exclusion (Fig. 6c). Multiple effects of virus-virus interactions on host ecology and evolution have been recently highlighted or proposed, and are the main focus 
of a nascent 'sociovirology' field ${ }^{39}$. Given their broad host range (Fig. 3), frequent detection alongside non-inovirus prophages (Supplementary Fig. 5), extended host cell residence time and the experimental results presented here, inoviruses could be driving many of these interactions and are undeniably important to consider in this framework.

\section{Discussion}

Taken together, the results presented here call for a complete re-evaluation of the diversity and role of inoviruses in nature. Collectively, inoviruses are distributed across all biomes and display an extremely broad host range spanning both prokaryotic domains of life. Comparative genomics revealed evidence of longstanding virus-host codiversification, leading to strong partitioning of inovirus diversity by host taxonomy, high inovirus prevalence in several microbial groups, including major pathogens, and potential interdomain transfer. Even though small $(5-20 \mathrm{~kb})$, their genomes encode a large functional diversity shaped by frequent gene exchange with unrelated groups of viruses, plasmids and transposable elements. Some of the many uncharacterized inovirus genes probably encode molecular mechanisms at the interface of virus-host and virusvirus interactions, such as modulators of the CRISPR-Cas systems, superinfection exclusion genes or toxin-antitoxin modules. This expanded and restructured catalogue of 5,964 distinct inovirus genomes thus provides a renewed framework for further investigation of the different effects that inoviruses have on microbial ecosystems, and exploration of their unique potential for biotechnological applications and manipulation of microorganisms.

\section{Methods}

Construction of an Inoviridae genome reference set. Genome sequences affiliated to Inoviridae and $\geq 2.5 \mathrm{~kb}$ were downloaded from NCBI Genbank and RefSeq on 14 July 2017 (refs. ${ }^{40,41}$ ). These were clustered at $98 \%$ ANI to remove duplicates and screened for cloning vectors and partial genomes (Supplementary Table 1). Two of these genomes (Stenotrophomonas phage phiSMA9, NC_007189, and Ralstonia phage RSS30, NC_021862) presented an unusually long section ( $\geq 1 \mathrm{~kb})$ without any predicted gene, associated with a lack of short genes that are typical of Inoviridae. For these, genes were predicted de novo using Glimmer ${ }^{42}$ trained on their host genomes (NC_010943 for phiSMA9 and NC_003295 for RSS30) with standard genetic code. Similarly, genes for Acholeplasma phage MV-L1 (NC_001341) were predicted de novo using Glimmer with genetic code 4 (Mycoplasma/Spiroplasma) and trained on the host genome (NC_010163), followed by a manual curation step to integrate both RefSeq-annotated genes and these newly predicted CDS.

Protein clusters (PCs) were computed from these genomes from an all-versusall blastp of predicted CDS (thresholds: $e \leq 0.001$, bit score $\geq 30$ ) and clustered with InfoMap ${ }^{33}$. Sequences from these PCs were then aligned with MUSCLE ${ }^{43}$, transformed into an HMM profile and compared with each other using $\mathrm{HHSearch}^{44}$ (cut-offs: probability $\geq 90 \%$ and coverage $\geq 50 \%$, or probability $\geq 99 \%$, coverage $\geq 20 \%$ and hit length $\geq 100$ ). The larger clusters generated through this second step are designated here as iPFs. Only ten PCs were clustered into larger iPFs, but these were consistent with the functional annotation of these proteins. For instance, one iPF combined two PCs both composed of replication initiation proteins.

Marker genes were identified from a bipartite network linking Inoviridae genomes to iPFs (Supplementary Fig. 1). Only the genes encoding the morphogenesis $(\mathrm{pI})$ protein represented good candidates for a universally conserved gene across all members of the Inoviridae, and HMM profiles were built for the three pI iPFs. To optimize these profiles, sequences were first clustered at $90 \%$ amino acid identity with cd-hit ${ }^{45}$, then aligned with MUSCLE ${ }^{43}$ and the profile generated with hmmbuild ${ }^{46}$

These reference genomes were also used to evaluate the detection of the Inoviridae structural proteins based on protein features beyond sequence similarity (see Supplementary Notes). Here, signal peptides were predicted using SignalP in both Gram-positive and Gram-negative modes $^{47}$, and TMDs were identified with $\mathrm{TMHMM}^{48}$

Search for inovirus in microbial genomes and metagenomes. Proteins predicted from 56,868 microbial genomes publicly available in the IMG as of October 2017 (Supplementary Table 2) were compared with the reference morphogenesis (pI) proteins with hmmsearch ${ }^{46}$ (hmmer.org, score $\geq 30$ and $e \leq 0.001$ ) for the pI-like iPFs and blastp ${ }^{49}$ (bit score $\geq 50$ ) for the singleton $\mathrm{pI}$ protein (Acholeplasma phage MV-L1). These included 54,405 bacterial genomes, 1,304 archaeal genomes and 1,149 plasmid sequences. A total of 6,819 hits were detected, from which 795 corresponded to complete inovirus genomes. These included 213 circular contigs, that is, likely complete genomes, and 582 integrated prophages with canonical attachment (att) sites, that is, direct repeats of $\geq 10 \mathrm{bp}$ in a tRNA or outside of an integrase gene. All sequences were manually inspected to verify that these were plausible inovirus genomes (see Supplementary Notes). The predicted pI proteins from the curated genomes were then added to the references to generate new improved HMM models. Using these improved models, an additional set of 639 putative $\mathrm{pI}$ proteins was identified. New models were built from these proteins and used in a third round of searches, which did not yield any additional genuine inovirus sequence after manual inspection.

An automatic classifier was trained on this extended inovirus genome catalogue, that is, the reference genomes and the 795 manually curated genomes, to detect putative inovirus fragments around pI-like genes, based on 10 distinctive features of inovirus genomes (Supplementary Fig. 2 and Supplementary Notes). These 795 manually curated genomes were identified from 17 host phyla (or class for Proteobacteria) and were later classified into 5 proposed families and 245 proposed subfamilies (see below 'Gene-content-based clustering of inovirus genomes'). Three types of classifiers were tested: random forest (function randomForest from $\mathrm{R}$ package randomForest ${ }^{50}$ using 2,000 trees, other parameters left as default), random forest with conditional inference (function cforest from $\mathrm{R}$ package party ${ }^{51}$ using 2,000 trees, other parameters left as default) and a generalized linear model with lasso regularization (function glmnet from $\mathrm{R}$ package glmnet ${ }^{52}$ ). The efficiency of classifiers was evaluated via a tenfold cross-validation in which the input data set was partitioned into ten equal-sized subsamples, with one retained for validation and the other nine used for training through the ten possible permutations. Results were visualized as a ROC curve generated with ggplot2 (refs. ${ }^{53,54}$ ). The importance of features in the random forest classifier was evaluated using the function 'importance', from the R package randomForest.

On the basis of the inflection point observed on the ROC curves, the random forest classifier was selected as the optimal method as it provided the highest truepositive rate $(>92 \%)$ for false-positive rates of $<1 \%$ (Supplementary Fig. 2). This model was then used to classify all putative inovirus fragments that had not been identified as complete genomes previously, using a sliding window approach (up to 30 genes around the putative $\mathrm{pI}$ protein), and looking for the fragment with the maximum score in the random forest model (if $>0.9$ ). For the predicted integrated prophages, putative non-canonical att sites were next searched as direct repeats (10 bp or longer) around the fragment. Overall, 3,908 additional putative inovirus sequences were detected, including 738 prophages flanked by direct repeats.

A similar approach was used to search for inovirus sequences in 6,412 metagenome assemblies (Supplementary Table 2). Predicted proteins were compared with the $4 \mathrm{HMM}$ profiles as well as to the Acholeplasma phage MV-L1 singleton sequence, which led to 27,037 putative pI proteins using the same thresholds as for isolate genomes. The final data set of inovirus sequences predicted from these metagenome assemblies consisted of 6,094 sequences, including 922 circular contigs, 44 prophages with canonical att sites (direct repeats of $10 \mathrm{bp}$ or longer in a tRNA or next to an integrase) and 994 prophages with non-canonical att sites (direct repeats of $10 \mathrm{bp}$ or longer).

Clustering of inovirus genomes in putative species. Next, we sought to cluster these putative inovirus genomes along with the previously collected reference genomes to remove duplicated sequences and to select only one representative per species. This clustering was conducted according to the latest guidelines submitted to the International Committee on Taxonomy of Viruses (ICTV) for Inoviridae, that is, "95\% DNA sequence identity as the criterion for demarcation of species" 5 (https://talk.ictvonline.org/files/ictv_official_taxonomy_updates_since_the_8th report/m/prokaryote-official/6774/download), and included our 10,295 sequences alongside the 56 reference genomes. Notably, however, predictions spanning multiple tandemly integrated inovirus prophages had to be processed separately, otherwise they could lead to clusters gathering multiple species. To detect these cases of tandem insertions, we searched for and clustered separately all predictions with multiple pI proteins, as this gene is expected to be present in single copy in inoviruses ( $n=800$ sequences)

All non-tandem sequences were first clustered incrementally with priority given to complete genomes over partial genomes as well as fragments identified in microbial genomes over fragments from metagenomes. First, circular contigs and prophages with canonical att sites identified in a microbial genome were clustered, and all other fragments were affiliated to these seed sequences. Next, unaffiliated fragments detected in microbial genomes and with non-canonical att sites (that is, simple direct repeat) were clustered together, and other fragments were affiliated to this second set of seed sequences. Finally, the remaining unaffiliated sequences detected in microbial genomes were clustered together. This allowed us to use the more 'certain' predictions (that is, circular sequences and prophages with identified att sites) preferentially as seeds of putative species.

A similar approach was used to cluster sequences identified from metagenomes, as well as to separately cluster putative tandem fragments, that is, those including multiple $\mathrm{pI}$ proteins. All the clustering and affiliation was done with a threshold of $95 \%$ ANI on $100 \%$ of alignment fraction (according to the ICTV guidelines), with sequence similarity computed using mummer ${ }^{56}$. 
Accumulation curves were calculated for 100 random ordering of input sequences using a custom perl script and plotted with ggplot2 (refs. ${ }^{53,54}$ ).

\section{Clustering of predicted proteins from non-redundant inovirus sequences.} Predicted proteins from the representative genome of each putative species were next clustered using the same approach as for the reference genomes. A clustering into PCs was first achieved through an all-versus-all blastp using hits with $e \leq 0.001$ and bit score $\geq 50$ or bit score $\geq 30$ if both proteins are $\leq 70$ amino acids. HMM profiles were constructed for the 5,142 PCs and these were compared all-versus-all using HHSearch, keeping hits with $\geq 90 \%$ probability and $\geq 50 \%$ coverage or $\geq 99 \%$ probability, $\geq 20 \%$ coverage and hit length of $\geq 100$. This resulted in 4,008 protein families (iPFs).

The PCs were subsequently used for taxonomic classification of the inovirus sequences (see below), while iPFs were primarily used for functional affiliation. iPF functions were predicted based on the affiliation of iPF members against PFAM v30 (score $\geq 30$ ), as well as manual inspection of individual iPFs using HHPred $^{57}$.

PCs containing $\mathrm{pI}$-like proteins were also further evaluated to identify potential false positives stemming from a related ATPase encoded by another type of virus or mobile genetic element (see Supplementary Notes). The criteria used to determine genuine inovirus pI-like PCs were: the PC members closest known functional domain was Zot (based on the hmmsearch against PFAM), the proteins contained one or two TMD (either N-terminal or C-terminal), at least half of the sequences encoding this PC also include other genes expected in an inovirus sequence such as replication initiation proteins, and no significant similarity could be identified to any other type of ATPase using HHpred $^{57}$.

Gene-content-based clustering of inovirus genomes. A bipartite network was built in which genomes and PCs (as nodes) are connected by an edge when a predicted protein from the genome is a member of the PC. This network was then used to classify inovirus sequences as done previously for dsDNA viruses ${ }^{32}$. PCs were used instead of iPFs as they offer a higher resolution. Sequences with two pI proteins (that is, tandem prophages) were excluded from this network-based classification as these could lead to improper connections between unrelated genomes. Singleton proteins were also excluded, and only PCs with at least 2 members were used to build the network. This network had a very low density $(0.05 \%)$ reflecting the fact that most PCs were restricted to a minor fraction of the genomes. Nevertheless, this type of network can still be organized into meaningful groups through information theoretic approaches: here, sequence clusters were obtained through InfoMap, with default parameters and a two-level clustering (that is, genomes can be associated with a group and a subgroup).

A summarized representation of the network was generated by displaying each subgroup (level 2) as a node with a size proportional to the number of species in the subgroup, and drawing an edge to a PC if $>50 \%$ of the subgroup sequences encode this PC, except for the larger group ('Protoinoviridae':Subfamily_1) where connections are drawn for PCs found in $>25 \%$ of the sequences. The network was then visualized using Cytoscape ${ }^{58}$, with nodes from the same group (level 1) first gathered manually, and nodes allotment within group automatically generated using Prefuse-directed layout (default spring length of 200).

To evaluate the taxonomic rank to which these groups and subgroups would correspond, we calculated pairwise amino acid identity percentage of pI proteins for genomes (1) between groups and (2) within groups but between subgroups, using Sequence Demarcation Tool ${ }^{59}$. These were then compared with the pairwise amino acid identity calculated with the same approach for established viral groups, namely, Caudovirales order using the terminase large subunit (TerL) as a marker protein, Microviridae using the major capsid protein (VP1) as a marker protein and Circoviridae using the replication initiation protein (Rep) as a marker protein (see Supplementary Notes).

Distribution of inovirus sequences by host and biome. The distribution of hosts for inovirus sequences was based on detections in IMG draft and complete genomes, that is, excluding all metagenome-derived detections but including detections in metagenome-assembled genomes (published draft genomes assembled from metagenomes). Host taxonomic classification was extracted from the IMG database. For visualization purposes, a set of 56 universal single-copy marker proteins ${ }^{60,61}$ was used to build phylogenetic trees for bacteria and archaea based on all available microbial genomes in $\mathrm{IMG}^{23}$ (genomes downloaded on 27 October 2017) and about 8,000 metagenome-assembled genomes from the Genome Taxonomy Database ${ }^{62}$ (downloaded on 18 October 2017). Marker proteins were identified with hmmsearch (version $3.1 \mathrm{~b} 2$, hmmer.org) using a specific HMM for each of the markers. Genomes lacking a substantial proportion of marker proteins $(>28)$ or which had additional copies of $>3$ single-copy markers were removed from the data set.

To reduce redundancy and to enable a representative taxon sampling, DNA directed RNA polymerase $\beta$-subunit $160 \mathrm{kDa}$ (COG0086) was identified using hmmsearch (hmmer 3.1b2) and the HMM of COG0086 (ref. ${ }^{63}$ ). Protein hits were then extracted and clustered with cd-hit ${ }^{45}$ at $65 \%$ sequence similarity, resulting in 99 archaeal and 837 bacterial clusters. Genomes with the greatest number of different marker proteins were selected as cluster representatives. For every marker protein, alignments were built with $\mathrm{MAFFT}^{64}$ (v7.294b) and subsequently trimmed with BMGE (v1.12) using BLOSUM30 (ref. ${ }^{65}$ ). Single-protein alignments were then concatenated, resulting in an alignment of 11,220 sites for the archaea and 16,562 sites for the bacteria. Maximum-likelihood phylogenies were inferred with FastTree2 (v2.1.9 SSE3, OpenMP) ${ }^{66}$ using the options: -spr 4 -mlacc 2 -slownni -lg.

A distribution of inovirus sequences across biomes was obtained by compiling ecosystems and sampling location of all metagenomes where at least one inovirus sequence was detected. This information was extracted from the GOLD database ${ }^{67}$, and the map was generated using the BaseMap functions from the matplotlib python library ${ }^{68}$

Estimation of inovirus prevalence and co-infection patterns. Prevalence and coinfection patterns were evaluated from the set of sequences identified in complete and draft microbial genomes from the IMG database, that is, excluding detections from metagenome assemblies. To control for the presence of near-identical genomes in the database, prevalence and co-infection frequencies were calculated after clustering host genomes based on pairwise ANI (cut-offs: 95\% nucleotide identity on $95 \%$ alignment fraction). Prevalence was calculated at the host genus rank as the number of genomes with one or more inovirus sequence detected. Cooccurrence of inoviruses was evaluated based on the detections of distinct species in single-host genomes. Finally, we evaluated the rate of bacteria and archaea co-infected by an inovirus and a member of the Caudovirales order, the group of dsDNA viruses including most of the characterized bacteriophages (both lytic and temperate) as well as several archaeoviruses. To identify Caudovirales infections, we used the gene encoding the terminase large subunit as a marker gene, and searched the same genomes from the IMG database for hits to the PFAM domains terminase_1, terminase_3, terminase_6 and terminase_GpA (hmmsearch, score $\geq 30$ ).

Phylogenetic trees of inovirus sequences. Phylogenies of inovirus sequences were based on multiple alignment of $\mathrm{pI}$ protein sequences. To obtain informative multiple alignments, an all-versus-all blastp ${ }^{49}$ of all $\mathrm{pI}$ proteins was computed and used to identify the nearest neighbours of sequences of interests. For sequences detected in archaeal genomes, an additional 10 most closely related sequences with $e \leq 0.001$, bit score $\geq 50$ and a blast hit covering $\geq 50 \%$ of the query sequence were recruited for each archaea-associated sequence to help populate the tree. A similar approach was used for the tree based on the integrase genes from archaea-associated inoviruses: the protein sequences for the three integrase genes were compared with the NCBI $n r$ database with blastp ${ }^{49}$ (bit score $\geq 50, e \leq 0.001$ ) to gather their closest neighbours across archaeal and bacterial genomes.

Resulting data sets were first filtered for partial sequences as follows: the average sequence length was calculated excluding the top and bottom $10 \%$, and all sequences shorter than half of this average were excluded. These protein sequences were next aligned with MUSCLE (v3.8.1551) ${ }^{43}$, automatically trimmed with trimAL (v1.4.rev15) ${ }^{69}$ (option gappyout), and trees were constructed using IQ-TREE (v1.5.5) with an automatic detection of optimal model ${ }^{70}$ and displayed using iToL ${ }^{71}$. The optimal substitution model, selected based on the Bayesian information criterion, was VT + F + R5 for the the pI phylogeny of archaeal inoviruses, and LG + R4 for the integrase phylogeny of archaeal inoviruses. Annotated trees are available at http://itol.embl.de/shared/Siroux (project 'Inovirus').

Functional affiliation of iPFs. An automatic functional affiliation of all iPFs was generated by compiling the annotation of all members based on a comparison to PFAM (data extracted from the IMG). To refine these annotations for functions of interest, namely, replication initiation proteins, integration proteins, DNA methylases and toxin-antitoxin systems, individual iPF alignments were submitted to the HHPred website ${ }^{57}$, and the alignments were visually inspected for conserved residues and/or motifs (Supplementary Table 5, motifs extracted from refs. ${ }^{72,73}$ and the PFAM database v30 (ref. $\left.{ }^{74}\right)$ ).

To identify toxin-antitoxin protein partners, all inovirus sequences were screened for co-occurring genes including an iPF annotated as toxin and/or antitoxin, and the list of putative pairs was next manually curated (Supplementary Table 5). This enabled the identification of putative antitoxin proteins detected as conserved uncharacterized iPF frequently observed next to a predicted toxin iPF.

Finally, putative structural proteins and DNA-interacting proteins were specifically searched for. Putative structural proteins were predicted as described above for the isolate reference genomes, that is, as sequences of 30-90 amino acids, after in silico removal of signal peptide, if detected, and displaying 1 or 2 TMD. For the most abundant iPFs predicted as major coat proteins, the secondary structure was predicted with Phyre2 (ref. ${ }^{75}$ ). For DNA-interacting proteins, PFAM annotations were screened for $\mathrm{HTH}, \mathrm{RHH}, \mathrm{Zn}$-binding and $\mathrm{Zn}$-ribbon domains. In addition, HHsearch was used to compare the iPFs to 3 conserved HTH domains from the SMART database ${ }^{76}:$ Bac_DnaA_C, HTH_DTXR and HTH_XRE (probability $\geq 90$ ).

CRISPR spacer matches and CRISPR-Cas systems identification. All inovirus sequences were compared with the IMG CRISPR spacer database with blastn, using options adapted for short sequences (-task blastn-short -evalue 1 -word_size 7 -gapopen 10 -gapextend 2 -penalty -1 -dust no). Only cases with zero or one mismatch were further considered. Next, the genome context of these spacers was 
explored to identify the ones with a clear associated CRISPR-Cas system and to affiliate these systems to the different types described. Only spacers for which a cas gene could be identified in a region of $\pm 10 \mathrm{~kb}$ were retained. The CRISPR-Cas system affiliation was based on the set of cas genes identified around the spacer and performed following the guidelines from ref. ${ }^{7}$

For host genomes with a self-targeting spacer, additional (that is, non-inovirus) prophages were detected using VirSorter ${ }^{20}$. The number of distinct prophages was also estimated using the detection of large terminase subunits (hmmsearch against PFAM database, score $\geq 30$ ). Putative Acr and anti-CRISPR-associated (Aca) proteins were first detected through similarity to previously described Acr systems $^{38}$ (blastp, $e \leq 0.001$ and score $\geq 50$ ). Putative Acr and Aca proteins were identified by searching for HTH-domain-containing proteins identified based on HTH domains in the SMART database (see above) in inovirus sequences displaying a match to a CRISPR spacer extracted from the same host genome.

Microscopy and PCR investigation of a predicted provirus in M.profundi MobM. M. profundi strain MobM cells were grown in anaerobic DSMZ medium 479 at $37^{\circ} \mathrm{C}$ with $5 \mathrm{mM}$ methanol added as a methanogenic substrate instead of trimethylamine $\mathrm{e}^{78}$. After $35 \mathrm{~h}$ of growth, anaerobic mitomycin $\mathrm{C}$ was added to the culture at a final concentration of $1.0 \mu \mathrm{g} \mathrm{ml}^{-1}$ to induce the provirus. Samples were collected before and $4 \mathrm{~h}$ after induction and were filtered with $0.22-\mu \mathrm{m}$ pore size polyethersulfone filters (Millipore, Fisher Scientific) to obtain a 'cellular' $(\geq 0.22 \mu \mathrm{m})$ and a 'viral' $(<0.22 \mu \mathrm{m})$ fraction

The four types of samples (with or without induction, cellular and viral fractions) were prepared and imaged at the Molecular and Cellular Imaging Center, Ohio State University, Wooster, OH, USA. An equal volume of $2 \times$ fixative (6\% glutaraldehyde and $2 \%$ paraformaldehyde in $0.1 \mathrm{M}$ potassium phosphate buffer $\mathrm{pH}$ 7.2) was added directly to the culture post-induction. Of the medium, $30 \mu \mathrm{l}$ was applied to a formovar and carbon-coated copper grid for $5 \mathrm{~min}$, blotted and then stained with $2 \%$ uranyl acetate for $1 \mathrm{~min}$. Samples were examined with a Hitachi H7500 electron microscope and imaged with the SIA-L12C (16 megapixels) digital camera.

PCRs were initially run for induced and non-induced samples on both size fractions with three pairs of primers: one internal to the predicted provirus (B primers), one spanning the insertion site ( $\mathrm{P}$ primers) and one spanning the junction of the predicted excised circular genome ( $\mathrm{C}$ primers). The reactions were conducted for 35 cycles with denaturation, annealing and extension cycles of 0.5 , 0.5 and $1.0 \mathrm{~min}$ at $95.0,52.0$ and $72.0^{\circ} \mathrm{C}$, respectively. For $\mathrm{C}$ primers, numerous nonspecific amplification products were obtained with these conditions, and another set of PCRs was conducted with higher annealing temperatures of $56.5^{\circ} \mathrm{C}$ and $57.5^{\circ} \mathrm{C}$, both in triplicates. The PCR product was then cleaned to remove polymerase, free dNTPs and primers (Zymo Research) and subsequently used as templates for Sanger sequencing. The resulting chromatograms were analysed using the $\mathrm{R}^{54}$ packages sangerseq $\mathrm{R}^{79}$, sangeranalyse $\mathrm{R}^{80}$ and readr ${ }^{81}$. The extracted primary sequences were aligned to the MobM genome using blastn $^{49}$ and MUSCLE ${ }^{43}$, and the alignment was visualized with Jalview ${ }^{82}$.

Experimental characterization of hypothetical proteins from self-targeted Pseudomonas inoviruses. Hypothetical proteins predicted on inovirus prophages, which were (1) found in Pseudomonas genomes, (2) predicted to be targeted by at least one CRISPR spacer from the same genome, and (3) for which no acr locus could be identified anywhere else in the same genome, were selected for further functional characterization. The ten candidate genes were first codon optimized for expression in Pseudomonas using an empirically derived codon usage table. Codon optimization and vendor defined synthesis constraints removal were performed using BOOST ${ }^{83}$. Synthetic DNA were obtained from Thermo Fisher Scientific and cloned in between the SacI and PstI sites of an Escherichia-Pseudomonas broad host range expression vector, $\mathrm{pHERD} 30 \mathrm{~T}^{84}$. All gene constructs were sequenceverified before testing.

P. aeruginosa strains (PAO1::pLac I-C CRISPR-Cas, PA14 and 4386) were cultured on LB agar or liquid media at $37^{\circ} \mathrm{C}$. The pHERD30T plasmids were electroporated into P.aeruginosa strains, and LB was supplemented with $50 \mu \mathrm{g} \mathrm{ml}^{-1}$ gentamicin to maintain the pHERD30T plasmid. Phages DMS3m, JBD30, D3, 14-1, Luz7 and KMV were amplified on PAO1, and phage JBD44a was amplified on PA14. All phages were stored in SM buffer at $4{ }^{\circ} \mathrm{C}$ in the presence of chloroform.

For phage titring, a bacterial lawn was first generated by spreading $6 \mathrm{ml}$ of top agar seeded with $200 \mu \mathrm{l}$ host bacteria on a LB agar plate supplemented with $10 \mathrm{mM} \mathrm{MgSO}_{4}, 50 \mu \mathrm{g} \mathrm{ml}^{-1}$ gentamicin and $0.1 \%$ arabinose. The I-C cas genes in strain PAO1 were induced with $1 \mathrm{mM}$ isopropyl- $\beta$-D-1-thiogalactopyranoside. Three microlitres of phage serially diluted in SM buffer was then spotted onto the lawn and incubated at $37^{\circ} \mathrm{C}$ for $16 \mathrm{~h}$. Growth rates were similar between cells transformed with an empty vector and cells transformed with a vector including a candidate gene, except for the two cases where no growth was observed after transformation (see Supplementary Notes).

Experimental confirmation of self-targeting lethality and trans-acting Acr activity from a co-infecting phage in a P. aeruginosa model. The effect of CRISPR targeting of an integrated inovirus prophage was assessed in the P. aeruginosa strain PA14, which naturally encodes an intact Pf1 inovirus prophage, and for which both natural CRISPR arrays were deleted (strain PA14 $\Delta$ CRISPR1/ $\Delta$ CRISPR2 (Pf1)). Host cells were transformed with plasmids encoding CRISPR spacers either targeting the Pfl coat gene or without a target in the host genome. To generate these plasmids, complementary single-stranded oligos (IDT) were annealed and ligated into a linearized derivative of shuttle vector pHERD30T bearing I-F direct repeats in the multiple cloning site downstream of the pBAD promoter. PA14 lysogens were electroporated with $100 \mathrm{ng}$ plasmid DNA, allowed to recover for $1 \mathrm{~h}$ in $\mathrm{LB}$ at $37^{\circ} \mathrm{C}$ and plated on $\mathrm{LB}$ agar plates supplemented with $50 \mu \mathrm{g} \mathrm{ml}^{-1}$ gentamicin and $0.1 \%$ arabinose. Colonies were enumerated after growth for $14 \mathrm{~h}$ at $37^{\circ} \mathrm{C}$. Transformation efficiency (TE) was calculated as colonies per microgram DNA, and the percentage TE was calculated by normalizing the TE of the CRISPR RNA-expressing plasmids to the TE of an empty vector.

To evaluate the effect of an acr locus from a co-infecting prophage on selftargeted inoviruses, strain PA14 $\Delta$ CRISPR1/DCRISPR2 (Pf1) was lysogenized with phage DMS3 $\mathrm{m}_{\text {acrIF } 1}$ by streaking out cells from a solid plate infection and screening for colonies resistant to superinfection by DMS3 $\mathrm{m}_{\mathrm{acrF}}$. Lysogeny was confirmed by prophage induction. The same plasmid transformation approach was then used to assess the effect of inovirus self-targeting on host cell viability.

Quantification and statistical analysis. Sequence similarity searches were conducted with thresholds of E-value $\leq 0.001$ and bit score $\geq 30$ or 50 , the former being used mainly for short proteins. The different classifiers (random forest, conditional random forest and generalized linear model) used to identify inovirus sequences were evaluated using a tenfold cross-validation approach. For all boxplots, the lower and upper hinges correspond to the first and third quartiles, respectively, and the whiskers extend no further than \pm 1.5 times the interquartile range.

Reporting Summary. Further information on research design is available in the Nature Research Reporting Summary linked to this article.

\section{Data availability}

The following files are available at https:/genome.jgi.doe.gov/portal/Inovirus/ Inovirus.home.html: Gb_files_inoviruses.zip: GenBank files of all representative genomes for each inovirus species; Ref_PCs_inoviruses.zip: PCs from the references (raw fasta, alignment fasta and $\mathrm{hmm}$ profile); iPFs_inoviruses. zip: protein families from the extended inovirus data set (raw fasta, alignment fasta and hmm profile); MobM_C_primer_amplicon.fasta: multiple sequence alignment of the $\mathrm{C}$ primer products with the Methanolobus MobM genome (NZ_FOUJ01000007), confirming that C primer products span the junction of the excised genome. Accession numbers of all inovirus sequences used as reference are listed in Supplementary Table 1. Accession numbers of all genomes and metagenomes mined, including detailed information for each (meta)genome in which some inovirus sequences were detected are available in Supplementary Table 2. Finally, the list of all inovirus genome accession numbers, along with taxonomic and environmental distribution information, is provided in Supplementary Table 3.

\section{Code availability}

The set of scripts and models used to detect inovirus sequences is available at https://bitbucket.org/srouxjgi/inovirus/src/master/Inovirus_detector/.

Received: 12 February 2019; Accepted: 5 June 2019; Published online: 22 July 2019

\section{References}

1. Rakonjac, J., Bennett, N. J., Spagnuolo, J., Gagic, D. \& Russel, M. Filamentous bacteriophage: biology, phage display and nanotechnology applications. Curr. Issues Mol. Biol. 13, 51-76 (2011).

2. Fauquet, C. M. The diversity of single stranded DNA. Virus Biodivers. 7, 38-44 (2006).

3. Marvin, D. A., Symmons, M. F. \& Straus, S. K. Structure and assembly of filamentous bacteriophages. Prog. Biophys. Mol. Biol. 114, 80-122 (2014).

4. Bradbury, A. R. M. \& Marks, J. D. Antibodies from phage antibody libraries. J. Immunol. Methods 290, 29-49 (2004).

5. Nam, K. T. et al. Stamped microbattery electrodes based on self-assembled M13 viruses. Proc. Natl Acad. Sci. USA 105, 17227-17231 (2008).

6. Ju, Z. \& Sun, W. Drug delivery vectors based on filamentous bacteriophages and phage-mimetic nanoparticles. Drug Deliv. 24, 1898-1908 (2017).

7. Henry, K. A., Arbabi-Ghahroudi, M. \& Scott, J. K. Beyond phage display: non-traditional applications of the filamentous bacteriophage as a vaccine carrier, therapeutic biologic, and bioconjugation scaffold. Front. Microbiol. 6, 755 (2015).

8. Ilyina, T. S. Filamentous bacteriophages and their role in the virulence and evolution of pathogenic bacteria. Mol. Genet. Microbiol. Virol. 30, 1-9 (2015).

9. Shapiro, J. W. \& Turner, P. E. Evolution of mutualism from parasitism in experimental virus populations. Evolution 72, 707-712 (2018).

10. Sweere, J. M. et al. Bacteriophage trigger anti-viral immunity and prevent clearance of bacterial infection. Science 363, eaat9691 (2019). 
11. Waldor, M. K. \& Mekalanos, J. J. Lysogenic conversion by a filamentous phage encoding cholera toxin. Science 272, 1910-1914 (1996).

12. Faruque, S. M. \& Mekalanos, J. J. Pathogenicity islands and phages in Vibrio cholerae evolution. Trends Microbiol. 11, 505-510 (2003).

13. Bille, E. et al. A virulence-associated filamentous bacteriophage of Neisseria meningitidis increases host-cell colonisation. PLoS Pathog. 13, e1006495 (2017)

14. Rice, S. A. et al. The biofilm life cycle and virulence of Pseudomonas aeruginosa are dependent on a filamentous prophage. ISME J. 3, 271-282 (2009).

15. Rakonjac, J. Filamentous bacteriophages: biology and applications. eLS https://doi.org/10.1002/9780470015902.a0000777 (2012).

16. Varani, A. M., Monteiro-Vitorello, C. B., Nakaya, H. I. \& Van Sluys, M.-A The role of prophage in plant-pathogenic bacteria. Annu. Rev. Phytopathol. 51, 429-451 (2013).

17. Mai-Prochnow, A. et al. 'Big things in small packages: the genetics of filamentous phage and effects on fitness of their host'. FEMS Microbiol. Rev. 39, 465-487 (2015).

18. Páez-Espino, D. et al. Uncovering Earth’s virome. Nature 536, 425-430 (2016).

19. Páez-Espino, D., Pavlopoulos, G. A., Ivanova, N. N. \& Kyrpides, N. C. Nontargeted virus sequence discovery pipeline and virus clustering for metagenomic data. Nat. Protoc. 12, 1673-1682 (2017)

20. Roux, S., Enault, F., Hurwitz, B. L. \& Sullivan, M. B. VirSorter: mining viral signal from microbial genomic data. PeerJ 3, e985 (2015).

21. Brum, J. R. \& Sullivan, M. B. Rising to the challenge: accelerated pace of discovery transforms marine virology. Nat. Rev. Microbiol. 13, 147-159 (2015).

22. Vega Thurber, R. V. et al. Laboratory procedures to generate viral metagenomes. Nat. Protoc. 4, 470-483 (2009).

23. Chen, I. M. A. et al. IMG/M: integrated genome and metagenome comparative data analysis system. Nucleic Acids Res. 45, D507-D516 (2017)

24. Kimura, M., Wang, G., Nakayama, N. \& Asakawa, S. in Biocommunication in Soil Microorganisms (ed. Witzany, G.) 189-213 (Springer, 2011).

25. Kim, A. Y. \& Blaschek, H. P. Isolation and characterization of a filamentous virus-like particle from Clostridium acetobutylicum NCIB-6444. J. Bacteriol. 173, 530-535 (1991)

26. Iranzo, J., Koonin, E. V., Prangishvili, D. \& Krupovic, M. Bipartite network analysis of the archaeal virosphere: evolutionary connections between viruses and capsid-less mobile elements. J. Virol. 90, 11043-11055 (2016).

27. Prangishvili, D., Bamford, D. H., Forterre, P. \& Iranzo, J. The enigmatic archaeal virosphere. Nat. Rev. Microbiol. 15, 724-739 (2017).

28. Krupovic, M., Cvirkaite-Krupovic, V., Iranzo, J., Prangishvili, D. \& Koonin, E. V. Viruses of archaea: structural, functional, environmental and evolutionary genomics. Virus Res. 244, 181-193 (2018).

29. Garushyants, S. K., Kazanov, M. D. \& Gelfand, M. S. Horizontal gene transfer and genome evolution in Methanosarcina. BMC Evol. Biol. 15, 102 (2015).

30. Mavrich, T. N. \& Hatfull, G. F. Bacteriophage evolution differs by host, lifestyle and genome. Nat. Microbiol. 2, 17112 (2017).

31. Krupovic, M., Prangishvili, D., Hendrix, R. W. \& Bamford, D. H. Genomics of bacterial and archaeal viruses: dynamics within the prokaryotic virosphere. Microbiol. Mol. Biol. Rev. 75, 610-635 (2011).

32. Iranzo, J., Krupovic, M. \& Koonin, E. V. The double-stranded DNA virosphere as a modular hierarchical network of gene sharing. $m$ Bio 7 , e00978-16 (2016).

33. Rosvall, M. \& Bergstrom, C. T. Multilevel compression of random walks on networks reveals hierarchical organization in large integrated systems. PLoS ONE 6, e18209 (2011).

34. Wolf, Y. I. et al. Origins and evolution of the global RNA virome. mBio 9, e02329-18 (2018).

35. Koonin, E. V., Dolja, V. V. \& Krupovic, M. Origins and evolution of viruses of eukaryotes: the ultimate modularity. Virology 479-480, 2-25 (2015).

36. Song, S. \& Wood, T. K. Post-segregational killing and phage inhibition are not mediated by cell death through toxin/antitoxin systems. Front. Microbiol. 9, 814 (2018)

37. Marraffini, L. A. CRISPR-Cas immunity in prokaryotes. Nature 526, 55-61 (2015)

38. Borges, A. L., Davidson, A. R. \& Bondy-Denomy, J. The discovery, mechanisms, and evolutionary impact of anti-CRISPRs. Annu. Rev. Virol. 4, 37-59 (2017).

39. Díaz-Muñoz, S. L., Sanjuán, R. \& West, S. Sociovirology: conflict, cooperation, and communication among viruses. Cell Host Microbe 22, 437-441 (2017).

40. O'Leary, N. A. et al. Reference sequence (RefSeq) database at NCBI: current status, taxonomic expansion, and functional annotation. Nucleic Acids Res. 44, D733-D745 (2016)

41. Brister, J. R., Ako-Adjei, D., Bao, Y. \& Blinkova, O. NCBI viral genomes resource. Nucleic Acids Res. 43, D571-D577 (2015).
42. Delcher, A. L., Bratke, K. A., Powers, E. C. \& Salzberg, S. L. Identifying bacterial genes and endosymbiont DNA with Glimmer. Bioinformatics 23, 673-679 (2007).

43. Edgar, R. C. MUSCLE: a multiple sequence alignment method with reduced time and space complexity. BMC Bioinformatics 5, 113 (2004).

44. Remmert, M., Biegert, A., Hauser, A. \& Söding, J. HHblits: lightning-fast iterative protein sequence searching by HMM-HMM alignment. Nat. Methods 9, 173-175 (2011)

45. Fu, L., Niu, B., Zhu, Z., Wu, S. \& Li, W. CD-HIT: accelerated for clustering the next-generation sequencing data. Bioinformatics 28, 3150-3152 (2012).

46. Eddy, S. R. Accelerated profile HMM searches. PLoS Comput. Biol. 7, e1002195 (2011)

47. Petersen, T. N., Brunak, S., Von Heijne, G. \& Nielsen, H. SignalP 4.0: discriminating signal peptides from transmembrane regions. Nat. Methods $\mathbf{8}$, 785-786 (2011).

48. Krogh, A., Larsson, B., von Heijne, G. \& Sonnhammer, E. L. L. Predicting transmembrane protein topology with a hidden Markov model: application to complete genomes. J. Mol. Biol. 305, 567-580 (2001).

49. Camacho, C. et al. BLAST+: architecture and applications. BMC Bioinformatics 10, 421 (2009).

50. Liaw, A. \& Wiener, M. Classification and regression by randomForest. $R$ News 2, 18-22 (2002)

51. Strobl, C., Boulesteix, A. L., Kneib, T., Augustin, T. \& Zeileis, A. Conditional variable importance for random forests. BMC Bioinformatics 9, 307 (2008).

52. Simon, N., Friedman, J., Hastie, T. \& Tibshirani, R. Regularization paths for Cox's proportional hazards model via coordinate descent. J. Stat. Softw. 39, 1-13 (2011).

53. Wickham, H. ggplot2: Elegant Graphics for Data Analysis (Springer, 2016).

54. R Core Team R: A Language and Environment for Statistical Computing (R Foundation for Statistical Computing, 2018).

55. Adriaenssens, E. M., Krupovic, M. \& Knezevic, P. Taxonomy of prokaryotic viruses: 2016 update from the ICTV bacterial and archaeal viruses subcommittee. Arch. Virol. 162, 1153-1157 (2017).

56. Kurtz, S. et al. Versatile and open software for comparing large genomes. Genome Biol. 5, R12 (2004).

57. Alva, V., Nam, S.-Z., Söding, J. \& Lupas, A. N. The MPI bioinformatics toolkit as an integrative platform for advanced protein sequence and structure analysis. Nucleic Acids Res. 44, W410-W415 (2016).

58. Demchak, B. et al. Cytoscape: the network visualization tool for GenomeSpace workflows. F1000Res. 3, 151 (2014).

59. Muhire, B. M., Varsani, A. \& Martin, D. P. SDT: a virus classification tool based on pairwise sequence alignment and identity calculation. PLOS ONE 9 , e108277 (2014)

60. Eloe-Fadrosh, E. A. et al. Global metagenomic survey reveals a new bacterial candidate phylum in geothermal springs. Nat. Commun. 7, 10476 (2016).

61. Yu, F. B. et al. Microfluidic-based mini-metagenomics enables discovery of novel microbial lineages from complex environmental samples. eLife 6, e26580 (2017)

62. Parks, D. H. et al. Recovery of nearly 8,000 metagenome-assembled genomes substantially expands the tree of life. Nat. Microbiol. 2, 1533-1542 (2017).

63. Tatusov, R. L. The COG database: a tool for genome-scale analysis of protein functions and evolution. Nucleic Acids Res. 28, 33-36 (2000).

64. Katoh, K. \& Standley, D. M. MAFFT multiple sequence alignment software version 7: improvements in performance and usability. Mol. Biol. Evol. 30 772-780 (2013)

65. Criscuolo, A. \& Gribaldo, S. BMGE (block mapping and gathering with entropy): a new software for selection of phylogenetic informative regions from multiple sequence alignments. BMC Evol. Biol. 10, 210 (2010).

66. Price, M. N., Dehal, P. S. \& Arkin, A. P. FastTree 2-approximately maximum-likelihood trees for large alignments. PLoS ONE 5, e9490 (2010).

67. Mukherjee, S. et al. Genomes Online Database (GOLD) v.6: data updates and feature enhancements. Nucleic Acids Res. 45, D446-D456 (2017).

68. Hunter, J. D. Matplotlib: a 2D graphics environment. Comput. Sci. Eng. 9, 90-95 (2007).

69. Capella-Gutiérrez, S., Silla-Martínez, J. M. \& Gabaldón, T. trimAl: a tool for automated alignment trimming in large-scale phylogenetic analyses. Bioinformatics 25, 1972-1973 (2009).

70. Nguyen, L. T., Schmidt, H. A., Von Haeseler, A. \& Minh, B. Q. IQ-TREE: a fast and effective stochastic algorithm for estimating maximum-likelihood phylogenies. Mol. Biol. Evol. 32, 268-274 (2015).

71. Letunic, I. \& Bork, P. Interactive Tree of Life (iTOL) v3: an online tool for the display and annotation of phylogenetic and other trees. Nucleic Acids Res. 44, W242-W245 (2016)

72. Krupovic, M. Networks of evolutionary interactions underlying the polyphyletic origin of ssDNA viruses. Curr. Opin. Virol. 3, 578-586 (2013).

73. Carr, S. B., Phillips, S. E. V. \& Thomas, C. D. Structures of replication initiation proteins from staphylococcal antibiotic resistance plasmids reveal protein asymmetry and flexibility are necessary for replication. Nucleic Acids Res. 44, 2417-2428 (2016). 
74. Finn, R. D. et al. The Pfam protein families database: towards a more sustainable future. Nucleic Acids Res. 44, D279-D285 (2016).

75. Kelley, L. A., Mezulis, S., Yates, C., Wass, M. \& Sternberg, M. The Phyre2 web portal for protein modelling, prediction, and analysis. Nat. Protoc. 10, 845-858 (2015).

76. Letunic, I. SMART 4.0: towards genomic data integration. Nucleic Acids Res. 32 142D-144D (2004).

77. Makarova, K. S. et al. An updated evolutionary classification of CRISPR-Cas systems. Nat. Rev. Microbiol. 13, 722-736 (2015).

78. Mochimaru, H. et al. Methanolobus profundi sp. nov., a methylotrophic methanogen isolated from deep subsurface sediments in a natural gas field. Int. J. Syst. Evol. Microbiol. 59, 714-718 (2009).

79. Hill, J. T. et al. Poly peak parser: method and software for identification of unknown indels using sanger sequencing of polymerase chain reaction products. Dev. Dyn. 43, 1632-1636 (2014).

80. Lanfear, R. sangeranalyseR: a suite of functions for the analysis of Sanger sequence data in R v.1.20.0 (2015).

81. Wickham, H., Hester, J. \& Francois, R. readr: read rectangular text data v.1.3.1 (2017).

82. Waterhouse, A. M., Procter, J. B., Martin, D. M. A., Clamp, M. \& Barton, G. J Jalview version 2-a multiple sequence alignment editor and analysis workbench. Bioinformatics 25, 1189-1191 (2009).

83. Oberortner, E., Cheng, J. F., Hillson, N. J. \& Deutsch, S. Streamlining the design-to-build transition with build-optimization software tools. ACS Synth. Biol. 6, 485-496 (2017).

84. Qiu, D., Damron, F. H., Mima, T., Schweizer, H. P. \& Yu, H. D. PBAD-based shuttle vectors for functional analysis of toxic and highly regulated genes in Pseudomonas and Burkholderia spp. and other bacteria.

Appl. Environ. Microbiol. 74, 7422-7426 (2008).

\section{Acknowledgements}

The MobM strain was provided by D. J. Ferguson, Miami University, Oxford, $\mathrm{OH}$, USA. Its genome was sequenced and assembled by the US Department of Energy Joint Genome Institute through a Community Science Program initiative to K.C.W. (CSP no. 1777). T. Meulia at the Molecular and Cellular Imaging Center, Ohio State University, Wooster, $\mathrm{OH}$, USA, performed the transmission electron microscopy of MobM samples. We gratefully acknowledge the contributions of many principal investigators who sent extracted DNA for isolate genome and metagenome sequencing as part of the Department of Energy Joint Genome Institute Community Science Program, and allowed us to include in our study the inovirus sequences detected in these publicly available data sets regardless of publication status (the complete list of data sets in which inovirus sequences were detected including principal investigators is available in Supplementary Table 2). This work was conducted by the US Department of Energy Joint Genome Institute, a Department of Energy Office of Science User Facility, under contract no. DE-AC02-05CH11231 and used resources of the National Energy Research Scientific Computing Center, which is supported by the Office of Science of the US Department of Energy under contract no. DE-AC02-05CH11231. R.A.D. and K.C.W. were partially supported by funding from the National Sciences Foundation Dimensions of Biodiversity (Award 1342701). M.K. was supported by l'Agence Nationale de la Recherche (France) project ENVIRA (ANR-17-CE15-0005-01). The Bondy-Denomy lab (A.L.B. and J.B.-D.) is supported by the UCSF Program for Breakthrough in Biomedical Research, funded in part by the Sandler Foundation, the NIH Office of the Director (DP5-OD021344) and NIGMS (R01GM127489). Research of P.B.M.C. was funded by the US Department of Energy award DE-AC02-05CH11231. Funding for A.S. was provided by the National Science Foundation grant EAR 1331940 (the Eel River Critical Zone Observatory).

\section{Author contributions}

S.R., M.K. and E.A.E.-F. conceived the study. R.A.D. and A.L.B. designed the archaeal inovirus induction and functional characterization of inovirus genes in Pseudomonas experiments, respectively. A.S. and P.B.M.C. contributed unpublished metagenomic data. S.R. and M.K. performed the data and metadata curation. S.R. developed the computational tools. S.N. and F.S. contributed additional computational analyses. R.A.D., J.-F.C. and A.L.B. performed the experiments. S.R., M.K., R.A.D., A.L.B., S.N., F.S., J.-F.C., N.N.I., J.B.-D., A.V., N.C.K. and E.A.E.-F. designed and wrote the manuscript. All authors reviewed and corrected the final manuscript.

\section{Competing interests}

The authors declare no competing interests.

\section{Additional information}

Supplementary information is available for this paper at https://doi.org/10.1038/ s41564-019-0510-x.

Reprints and permissions information is available at www.nature.com/reprints. Correspondence and requests for materials should be addressed to S.R. or E.A.E. Publisher's note: Springer Nature remains neutral with regard to jurisdictional claims in published maps and institutional affiliations.

This is a U.S. government work and not under copyright protection in the U.S.; foreign copyright protection may apply 2019

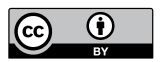

Open Access This article is licensed under a Creative Commons Attribution 4.0 International License, which permits use, sharing, adaptation, distribution and reproduction in any medium or format, as long as you give appropriate credit to the original author(s) and the source, provide a link to the Creative Commons license, and indicate if changes were made. The images or other third party material in this article are included in the article's Creative Commons license, unless indicated otherwise in a credit line to the material. If material is not included in the article's Creative Commons license and your intended use is not permitted by statutory regulation or exceeds the permitted use, you will need to obtain permission directly from the copyright holder. To view a copy of this license, visit http://creativecommons.org/licenses/by/4.0/. 


\title{
natureresearch
}

\section{Reporting Summary}

Nature Research wishes to improve the reproducibility of the work that we publish. This form provides structure for consistency and transparency in reporting. For further information on Nature Research policies, see Authors \& Referees and the Editorial Policy Checklist.

\section{Statistics}

For all statistical analyses, confirm that the following items are present in the figure legend, table legend, main text, or Methods section.

n/a Confirmed

Х $\square$ The exact sample size $(n)$ for each experimental group/condition, given as a discrete number and unit of measurement

Х $\square$ A statement on whether measurements were taken from distinct samples or whether the same sample was measured repeatedly

$\square$ The statistical test(s) used AND whether they are one- or two-sided

Only common tests should be described solely by name; describe more complex techniques in the Methods section.

$\square$ \A description of all covariates tested

Х $\square$ A description of any assumptions or corrections, such as tests of normality and adjustment for multiple comparisons

$\triangle \square$ A full description of the statistical parameters including central tendency (e.g. means) or other basic estimates (e.g. regression coefficient)

AND variation (e.g. standard deviation) or associated estimates of uncertainty (e.g. confidence intervals)

$\square$ For null hypothesis testing, the test statistic (e.g. $F, t, r$ ) with confidence intervals, effect sizes, degrees of freedom and $P$ value noted

Give P values as exact values whenever suitable.

Х $\square$ For Bayesian analysis, information on the choice of priors and Markov chain Monte Carlo settings

Х $\square$ For hierarchical and complex designs, identification of the appropriate level for tests and full reporting of outcomes

$\square$ ¿stimates of effect sizes (e.g. Cohen's d, Pearson's $r$ ), indicating how they were calculated

Our web collection on statistics for biologists contains articles on many of the points above.

\section{Software and code}

Policy information about availability of computer code

Data collection Data were collected using a custom set of scripts specifically designed to identify inovirus genomes. These are available at https:// github.com/simroux/Inovirus/tree/master/Inovirus_detector

Data analysis

\begin{abstract}
For specific reference inoviruses, genes were predicted de novo using Glimmer v3. Sequence similarity searches were conducted using blast+ v2.7.1, hmmer 3.1b2, hhpred (online at https://toolkit.tuebingen.mpg.de/\#/tools/hhpred) and hhsearch v2.0.15. Sequences were clustered using InfoMap 0.18.25, mummer 3.0, SDT 1.0, and cd-hit 4.7. Viral sequences (non-inoviruses) were automatically detected using VirSorter v1.0.5. Signal peptide and transmembrane domains were predicted using SignalP 4.1 and TMHMM 2.0c. Trees were built using FastTree2 and IQ-Tree 1.5.5, based on alignments computed with muscle 3.8 or MAFFT v7.294b and automatically trimmed with trimAL v1.4 or BMGE v1.12. Alignments were manually inspected using Jalview v10.0.2. Statistical analyses, sanger sequenced reads interpretation, and automatic classifier design were conducted in R 3.4.1, using the following packages: randomForest, party, g|mnet, sangerseqR, sangeranalyseR, and readR. Secondary structure of putative inovirus major capsid proteins were predicted using Phyre v2.0. Figures were generated with R 3.4.1 using the ggplot2 package, Cytoscape v3.6.1, iTOL v4.4.1, and python v3.6.2 using matplotlib v2.0.2. Constraints in sequences to be synthesized were automatically identified and adjusted using BOOST v1.3.3.
\end{abstract}

For manuscripts utilizing custom algorithms or software that are central to the research but not yet described in published literature, software must be made available to editors/reviewers. We strongly encourage code deposition in a community repository (e.g. GitHub). See the Nature Research guidelines for submitting code \& software for further information.

\section{Data}

Policy information about availability of data

All manuscripts must include a data availability statement. This statement should provide the following information, where applicable:

- Accession codes, unique identifiers, or web links for publicly available datasets

- A list of figures that have associated raw data

- A description of any restrictions on data availability 


\section{Field-specific reporting}

Please select the one below that is the best fit for your research. If you are not sure, read the appropriate sections before making your selection. \Life sciences Behavioural \& social sciences Ecological, evolutionary \& environmental sciences

For a reference copy of the document with all sections, see nature.com/documents/nr-reporting-summary-flat.pdf

\section{Life sciences study design}

All studies must disclose on these points even when the disclosure is negative.
Sample size
No sample size calculation was performed, as the largest collection of publicly available data possible was mined.
Data exclusions
No data were excluded.
Replication
None of the findings was found to be impossible to replicate. This includes PCR amplification of the putative archaeal inovirus provirus, which was repeated either two of three times with similar results (see Supplementary Fig. 11), and the superinfection experiments which were conducted twice and produced similar results.
Randomization None of the analyses involved allocation of samples to different groups.
Blinding
None of the analyses required blind investigation since the study does not involve a treatment vs control trial (with the exception of "obvious" negative controls such as "no template" PCR).

\section{Reporting for specific materials, systems and methods}

We require information from authors about some types of materials, experimental systems and methods used in many studies. Here, indicate whether each material, system or method listed is relevant to your study. If you are not sure if a list item applies to your research, read the appropriate section before selecting a response.

\begin{tabular}{l} 
Materials \& experimental syste \\
\hline n/a
\end{tabular}

Methods $\mathrm{n} / \mathrm{a}$ Involved in the study

\ $\square$ ChIP-seq

Х $\square$ Flow cytometry

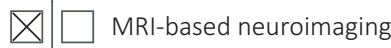

